\title{
An Information Retrieval Approach for Automatically Constructing Software Libraries
}

\author{
Yoëlle S. Maarek \\ IBM Thomas J. Watson Research Center \\ P.O. Box 704 \\ Yorktown Heights, NY 10598 \\ yoelle@ibm.com \\ Daniel M. Berry' \\ Technion, Israel Institute of Technology \\ Computer Science Department \\ Haifa, 32000, Israel \\ dberry@techsel.bitnet \\ Gail E. Kaiser \\ Columbia University \\ Department of Computer Science \\ New York, NY 10027 \\ kaiser@cs.columbia.edu
}

September 1990

CUCS-049-90

(C)1990 Yoëlle S. Maarek, Daniel M. Berry and Gail E. Kaiser. 


\title{
An Information Retrieval Approach for Automatically Constructing Software Libraries
}

\author{
Yoëlle S. Maarek \\ IBM Thomas J. Watson Research Center \\ P.O. Box 704 \\ Yorktown Heights, NY 10598 \\ ynelle@ibm.com
}

\author{
Daniel M. Berry \\ Technion, Israel Institute of Technology \\ Computer Science Department \\ Haifa, 32000, Israel \\ dberry@techsel.bitnet
}

\author{
Gail E. Kaiser \\ Columbia University \\ Department of Computer Science \\ New York, NY 10027 \\ kaiser@cs.columbia.edu
}

\begin{abstract}
Although software reuse presents clear advantages for programmer productivity and code reliability, it is not practiced enough. One of the reasons for the only moderate success of reuse is the lack of software libraries that facilitate the actual locating and understanding of reusable components. This paper describes a technology for automatically assembling large software libraries that promote software reuse hy helping the user locate the components closest. to her/his needs.

Software libraries are automatically assembled from a set of unorganized components by using information retrieval techniques. The construction of the library is done in liwn steps. First, attributes are automatically extracted from natural language documentation by using a new indexing scheme based on the notinns of lexical affinities and quantity of information. Then, a hierarchy for browsing is automatically generated using a clustering technique that iraws only on the information provided by the attributes. Thanks to the free-text indexing scheme, tnols following this apprnach can accept free-style natural language queries.

This technology has been implemented in the Gunu system, which has been applied to construct an organized library of AIX utilities. An experiment was rondurted in order in evaluate the retrieval effectiveness of GURU as compared to infoF,XPLORER a hypertext lihrary system for Aix 3 nn the IBM RISC System/6000 series. We followed the usual evaluation prowerlure used in information retrieval, hased upon recall and precision measures, and determined that. nur system performs $15 \%$ hetter on a random test set, while being much less expensive to build than InfoFirlorfn.
\end{abstract}

Index Terms: autnmatic indexing, clustering, information retrieval, lexical affinities, snftware libraries, soltware reuse. 


\section{Introduction}

Software reuse is widely believed to be a promising means for improving software productivity and reliability [14], and therefore is an issue of growing interest in software engineering. Unfortunately, not enough adequate libraries of reusable software components are available. By adequate, we mean that the library:

- provides a sufficient number of components, over a spectrum of domains, that can be reused as is (black-box reuse) or easily adapted (white-box reuse), and

- is organized such that existing code closest to the users' needs is easy to locate. In particular. the library should provide mechanisms to help the reuser look for "functionally close" components that meet some given requirements.

This paper is concerned with the second adequacy issue, and more generally with library systems that. provide means for representing, storing and retrieving relssable components.

The first. stage in building a library consists of indexing the objects to be stored in it. that is, producing a set of characterizing attributes, or signature, for each of these objects. The signatnre for each object represents the reusable object. Therefore. the quality of indexing is crucial to the quality of the library. Functionality is an important aspect of software components. Thus, it is necessary to include conceptual information about functionality in the indices. Unfortunately, conceptual information is difficult to obtain. Few programmers provide conceptual indices for their code. Moreover, even if provided, they can hardly be expressed under a common formalism since pieces of code typically originate from multiple sources. One solution is to manually index software components a posteriori according to a given classifying scheme. but this task is both arbitrary and tedious.

As an alternative, we propose to automatically identify indices by analyzing the natural-language dorumentation, in the form of manual pages or comments, usually associated with the code. Naturallanguage documentation is clearly a rich source of conceptual information. However, this information is contained only implicitly, in an unstructured way, and is not usable as such. In order to extract usable information from free-style documentation, we propose to use information retrieval techniques. Once the indices have been produced, components can be automatically classified, stored and retrieved according to their signatures.

The classifying stage in the construction of a library consists of gathering objects into classes such that the members of the same class share some set of properties. The basic motivation for classifying is to facilitate browsing among similar components in order to identify the best candidates for reuse. So that. during retrieval, a set of potentially adaptable components can be easily located. Browsing is more important for software libraries than for other kind of libraries, since there rarely exists a component perfectly matching a user's query. Moreover, local browsing allows the user to discover unanticipated opportunities for reuse.

We have designed and implemented a tool, Guru, that embodies the above approach. Guru automatically assembles conceptually structured software libraries from a set of unindexed and unorganized software components. In the first stage. Gunu extracts the indices from the natural language documentation associated with the software components to be stored, by using a new indexing scheme. This indexing scheme is based on lexical affinities and on their statistical distribution. It identifies a set of attributes for each document to represent a functional description of the associated software unit. In the second stage, GuRU assembles the indexed objects into a browsing hierarchy by using a hierarchical clustering technique that draws information exclusively from the indices identified in the previous stage. Thus, Guru 
supports both classical linear retrieval, in which candidates are ranked arcording to a numerical measure that evaluates how well they answer the query, and cluster-based retrieval in which the browse hierarchy directs the search for the best candidate.

Section 2 brief y compares the artificial intelligence and information retrieval approaches to construction of software libraries and explains why we follow an IR approach. Section 3 describes the indexing method. Section 4 presents the classification approach and the clustering technique used for assembling the library. Section 5 deals with the retrieval stage. Section 6 gives results using our GuJu implementation and a formal evaluation based on usual methodology for evaluating information retrieval systems. Finally. Section 7 summarizes the main contributions of this work. Related work is discussed as relevant throughout the paper.

\section{AI vs IR approach}

Previous efforts for building reuse systems can be roughly classified into two groups according to the approach adopted, the information retrieval (IR) approach or the artificial intelligence (AI) approach.

The IR approach consists of drawing information only from the structure of some documents that provide information on the software components. No semantic knowledge is used and no interpretation of the document is given: the reuse tool attempts to characterize the document rather than understand it. There are currently very few software library systems that follow an IR approach, or use existing IR techniques. Among them, the RsL system, [6] for instance, automatically scans source code files and extracts comments explicitly labeled for reuse with attributes such as keyword, author, date created, etc. The keyword attribute provides a list of free-text single-term indices very much like those used in IR tools. The Reuse system [3] provides a menu-driven front end to an information retrieval system, thus all kind of software objects (including user menus and system thesauri) are stored as textual documents. Thus, the two previous systems use some kind of IR related technique, however the only system, to our knowledge, that applies a pure IR approach is the system proposed by Frakes and Nejmeh [15]. They use the CATALOG information retrieval system for storing and retrieving $\mathrm{C}$ software components. Each component is characterized by a set of single-term indices that are allomatically extracted from the natural-language headers of $\mathrm{C}$ programs. Therefore, the construction of the $\mathrm{C}$ components repository is done antomatically, and does not require any pre-encoded knowledge as in RsL for instance.

In contrast. in the $\Lambda I$ approach, the rense tool aims at understanding the queries and the functionality of components before providing an answer. AI-based systems are often smarter than IR systems. Some of them are context sensitive and can generate answers adapted to the user's expertise. As a t,radeoff, they require some domain analysis and a great deal of pre-encoded semantic information, which is usually providerl manually. They are based upon a knowledge base that stores semantic information about the domain and about the language itself in case of a natural-language interfare. The main problem of applying this approach in the context of software libraries is that many domains cannot be easily circumscribed and the domain analysis is very difficult [10]. This makes the construction of such systems very tedious and expensive. Examples of AI or knowledge-based rense tools are numerous. e.g.. [30], [39], [2], [11], [37].

The AI approach can be useful in some applications. However, we prefer the IR approach for reasons of

- cost: the library system is built entirely antomatically,

- transportability: the library system can be rebuilt for any domain since it does require manually 
provided domain knowledge,

- scalability: the repository can be easily updated when new components are inserted, either by recompiling the indices or by applying incrementaj techniques, the indexing task is entirely mechanical.

We therefore propose to apply a pure IR approach, in the same direction of research as Frakes and Nejmeh, by automatically bnilding free-text indices that characterize soltware components. We also propose to use an indexing scheme richer than the single-term indexing used in the IR-based tools described in this section so as to achieve a better retrieval effectiveness. The following section explains our sonrce of information and how the indexing is performed.

\section{The Indexing Stage}

The major advantage of automatic indexing over manual indexing, besides the obvious cost considerations, is that it allows a unified scheme, insuring that indices will be compatible with each other. The idea is to extract attribntes from an existing sonrce of information, i.e., the code and the natural-langnage documentation. Some work has been done towards extraction of primitive functional information from the code [26]. [34], however, the richer source of functional information is the natural-language documentation, assuming any is available.

An examination of numerous samples of code allowed us to reach the conclusion that some useful information can be ext,racted from programs written in a high-level language using good programming style, whereas little conceptual information can be found in typical real-world code chosen at random [24]. Unfortunately, even when dealing with well-written code, there is a very low probability that the programming styles of the various pieces of code will be consistent. Even a single programmer may use totally different identifiers for expressing the same concept from one day to another. Since software components come from multiple sources in the context of large software libraries, extracting attributes from code would necessitate as many indexing schemes as there are code sources. Another limitation comes from the fact that there are many more possibilities for identifers than for natural-language words since they do not follow any morphological or syntactic rules.

In other words, when there is no way to guarantee good, and let alone consistent and compatible, programming styles, extracting attributes from raw code does not give significant results. Therefore, we prefer concentrating on the other possible source of information, i.e., the natnral-language documentation either inserted into the code, i.e., the comments, or associated with the code, e.g., manual pages.

Comments are intended to help programmers understand the conde and thus may provide functional information. They deal with specific parts of the code into which they are inserted, and they may give information on varions parts at various levels of abstrartion. Fxtracting functional information from comments entails two activities,

- defining an indexing scheme that allows extracting attributes from natural language phrases or sentences. and

- relating comments to the portion of code they concern.

The second activity is very complex in free-style code. Indeed, in free-style programming, programmers can insert comments wherever, and in any format and any length, they wish. Although comments usually describe the containing routine or the one just below, in general it is impossible to automatically determine 
what part of the code is covered. A solntion wonld be to consider that all the comments inserted in a specific piece of code constitute a global natural-language description of the considered code. Unfortunately, this is not the case. Comments rank from low-level implementation details to high-level description. For instance, in the Im. $c$ source file in Berkeley UNIX, one can find comments as varions as:

1* current pointer to end of path $* /$, or

1* rm - for ReMoving files, directories trees. *I

The first conveys no 1 seful functional information while the second hits the mark exactly. In general, there are many more low level. and nseless for our purpose. comments than high level ones. and there is no way to antomatically distinguish between them. Therefore, so long as no style is enforced, it is very difficult to extract useful information from comments.

Let, us note, however, that any piece of natural language, from comments inserted in the code to design specifications, which is specifically related to software code and whose level of abstraction is known can bring nseful information. Thus, we are currently working on extracting functional information from comments in the framework of RPDE [17], a structured software development environment. in which comments are linked to the portion of code they describe. In the following, though, we try to remain as general as possible. and we do not assume that any commenting style is enforced. Therefore, although our indexing scheme is applicable to any piece of natural-language that brings some functional information, we will exemplify it through the analysis of manual pages clearly related to reusable components, such as UNIX-like manual pages.

In the rest of this paper, the AIX documentation is taken as our corpus since it fulfills the requirement of being structured into manual pages. Moreover the AIX documentation can be seen as a regular realworld documentation database since it is of average quality as far as commenting style is concerned. Many even consider the AIX documentation of poor quality when compared to Berkeley UNIX documentation due to typos. inconsistent style, poor vocabulary, etc.

\subsection{A Richer Indexing Unit: the Lexical Affinity}

There has been much work in IR dealing with natural-language text, a large variety of techniques have been devised for indexing. classifying and retrieving documents [31]. One of the main concerns in IR is the antomatic indexing of documents, which consists of producing for each document, a set of indices that form a signature of the document. A signature is a short-form description of a document, easier to manipulate than the entire document, which plays the role of a surrogate at the retrieval stage.

Several issues need to be addressed when indexing a docmment with respect to the natnre and the form of the produced indices. More precisely, the indexing vocabulary can be either controlled or uncontrolled. In the controlled vocabulary approach only a restricted set of indices are anthorized (e.g., in MEDLARS [32]), whereas in the uncontrolled vocabulary, or free text, approach, there is no constraint on the nature of the indices. It has been shown that both approaches are comparable in terms of performance, [14], [32], however we prefer the incontrolled vocabulary approach in the context of software reuse. for the same reasons of cost, portability and scalability. Indeed. defining an adequate controlled vocabulary is a mannal, domain-dependent task and. therefore, suffers from the same drawbacks as the encoding of a knowledge-base.

Another important issue in automatic indexing deals with the nature of the indices. The most usual form is single-term index. in which single words without contextual information are selected as indices. Unfortunately, single term indices are often too specific or too broad and can induce ambiguities. Therefore, 
it has been proposed to take term phrases as indexing units rather than single terms so as to refine the meaning of constituent words. However. the use of word co-occurrences has not brought good results as expressed by Salton [31] (p 296):

“... a phrase-formation process controlled only by word co-occurrences and the document frequencies of certain words is not likely to generate a large number of high-quality phrases."

As an answer to this problem, a possible solution has been to add syntactic criteria in order to provide further control in phrase formation, such as part-of-speech using specially formatted dictionaries [21]. or more refined analysis including semantics [36]. But,

"The available options in phrase generation appear limited, and the introduction of costly and refined methodologies may bring only marginal improvements." [31] (p 298)

We are more optimistic, and believe that indexing units richer than single terms can be used and bring significant improvement at low cost. The atomic unit we propose to use in order to demonstrate this is derived from the notion of lexical affinity. In linguistics, a syntagmatic lexical affinity (LA), also termed lexical relation, between two units of language slands for a correlation of their common appearance in the utterances of the language [8]. The observation of LAs in large textual corpora has been shown to convey information on both syntactic and semantic levels, and provides "Is with a powerful way of taking context in to account [35].

We propose to use the notion of LA for indexing purposes, and restrict the above definition by observing LAs within a finite document rather than within the whole language so as to retrieve conceptual affinities that characterize the document ${ }^{1}$, rather than purely lexical ones. Moreover, we only consider LAs involving open-class words as meaning-bearing, whereas LAs involving closed-class words ${ }^{2}$ are not.

Ideally. LAs are extracted from a text by parsing it since two words share a lexical affinity if they are involved in a modifier-modified relation. Unfortunately, automatic syntactir parsing of free-style text is still not very efficient [33]. Instead, we make use of simple co-occurrence. It has been shown by Martin et al. that $98 \%$ of lexical relations relate words that are separated by at most five words within a single sentence [28]. Therefore, most of the LAs involving a word $w$ can be extracted by examining the neighborhood of each occurrence of $u$ within a span of five words $(-5$ words and +5 words around $w)$.

The extraction technique consists of sliding a window over the text and storing pairs of words involving the head of the window (if it is an open-class word) and any of the other open-rlass elements of the window. The window is slid word by word from the first word of the sentence to the last, the size of the window decreasing at the end of the sentence so as not to cross sentence houndarins ${ }^{3}$. since lexical affinities cannot relate words belonging to different sentences. The window size being smaller than a ronstant, the extraction of LAs is linear in the number of words in the document. An algorithm for the sliding window technique is presented in Fignre 1. Maarek and Smadja have nsed a similar technirjue in [27], which was also based on Martin's results [28], but more adapted to the analysis of large corpora.

In summary, the first stage in indexing a manual page consists of extracting all the potential LAs by using the sliding window technique, and storing them under their canonical form, in which each word is represented by its inflectional root (or lemma). An example of the potential LAs extracted from the

\footnotetext{
'rathet than the whole language.

${ }^{2}$ In general, open-class words include nouns, verbs, adjectives and adverbs, while elosed-class words are pronouns, prepositions, conjunctions and interjections.

${ }^{3}$ The isolation of sentences is the only parsing performed
} 


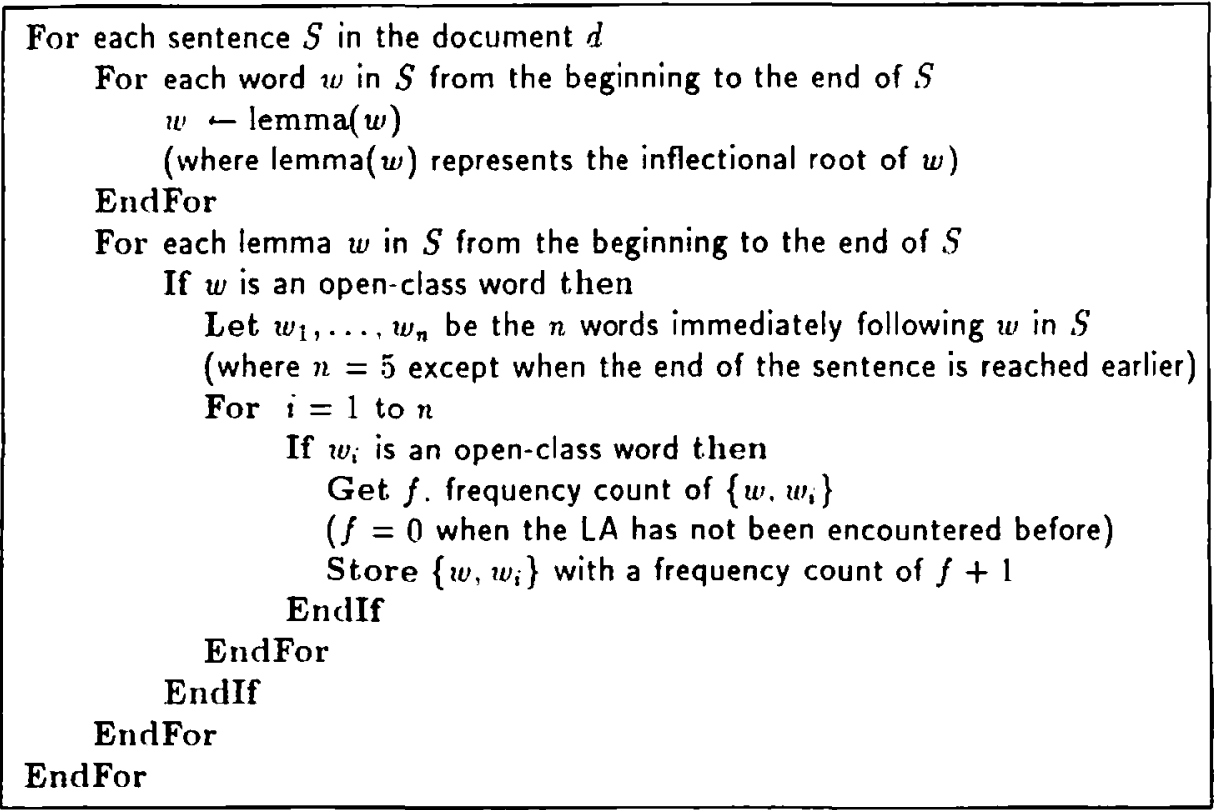

Figure 1: Sliding window technique

manual page of $\mathrm{mv}$ in $\Lambda \mathrm{IX}$ and ranked by frequency of occurrence are presented in Table 1 . For the sake of the comparison, a list of the single words extracted from the same manual page is shown in the first column, also ranked by frequency of appearance.

$\Lambda$ mong the extracted lexical relations, some correspond to abstractions of the considered document. and some do not. Since we are interested in indexing textual documents, in the first stage. we isolate actual affinities by using frequency criteria. It has been demonstrated that the frequency of occurrence of a term within a document is related to the importance of the word in a text [23]. This is also true for the common appearance of pairs of words and even more for lexical affinities.

\subsection{From LAs to Indices}

When analyzing a document. many potential lexical affinities are thus identified. Some of these lexical affinities are conceptually important and some are not. As seen in Tahle 1, frequency of appearance is a gond indicator of relevance. However, some noise exists, mainly due to worrs appearing ton often in a given context. In order to reduce the influence of such words, it is neressary in the second stage to select from among the lexical affinities identified only the most representative ones. i.e., those containing the most information.

We have defined a measure evaluating the resolving power of an LA. It is based upon the quantity of information of each of the words involved in the LA, as well as "pon the frequency of appearance of this LA within the considered document. The quantity of information of a word within a corpus is defined as:

$$
\operatorname{INFO}(w)=-\log _{2}(P\{w\})
$$

where $P\{w\}$ is the observed probability of occurrence $w$ in the corpus [4]. [32]. Therefore, the more 


\begin{tabular}{|l|l||l|l|}
\hline open-class words & freq & LAs & freq \\
\hline file & 30 & file move & 9 \\
directory & 14 & be fle & 8 \\
mv & 11 & directory file & 7 \\
files & 8 & file system & 5 \\
new & 7 & file overwrite & 5 \\
name & 7 & file mv & 5 \\
move & 7 & file name & 4 \\
newname & 6 & name path & 3 \\
is & 6 & do file & 3 \\
system & 5 & directory move & 3 \\
one & 5 & different file & 3 \\
$\ldots$ & $\ldots$ & $\ldots$ & $\ldots$ \\
\hline
\end{tabular}

Table 1: Keywords and lexical affinities classified by frequency in the mv manual page

frequent a word is in a domain, the less information it carries. From this definition, we infer the definition of the quantity of information of an LA $\left(w_{1}, w_{2}\right)$ as:

$$
\operatorname{INFO}\left(\left(w_{1}, w_{2}\right)\right)=-\log _{2}\left(P\left\{w i, w_{2}\right\}\right)
$$

To simplify the computation of this factor, in the rest of this work, we consider words within the textual universe as independent variables ${ }^{4}$. Thus, we use the following formula for computing the quantity of information of an LA.

$$
\operatorname{INFO}\left(\left(w_{1}, w_{2}\right)\right)=-\log _{2}\left(P\left\{w_{1}\right\} \times P\left\{w_{2}\right\}\right)
$$

Then, we define the resolving power of an LA in a given document as follows. Iet $\left(u^{\prime \prime}, w_{2}, f\right)$ be a tuple retrieved while analyzing a document $d$, where $\left(w_{1}, w_{2}\right)$ is an $L \Lambda$ appearing $f$ times in $d$. The resolving powers of this $L A$ in $d$ is defined as:

$$
\rho\left(\left(w_{1}, w_{2}, f\right)\right)=f \times \operatorname{INFO}\left(\left(w_{1}, w_{2}\right)\right)
$$

The higher the resolving power of a lexical affinity is, the more characteristic of the document it is. The resolving power allows us to evaluate the importance of a lexical affinity within a text by taking into account both its frequency of appearance in the text and the quantity of information of the words involved. Thus, even though the lexical affinity (be file) appears very often in an AIX manual page. it has only a small resolving power, simply because the quantity of information of hoth the words "file" and "be" in the AIX documentation is low.

In order to be able to compare the relative performances, in terms of resolving power, of different documents, we transform the raw $\rho$ score into a standardized score. The standardized score, or z-score, is defined as $\rho_{z}=(\rho-\bar{\rho}) / \sigma$ where $\bar{\rho}$ and $\sigma$ are the average and standard deviation of the $\rho$-values. This transformation does not alter the distribution and allows us to evaluate the relative status of the score in the $\rho$ distribution. In the rest of this paper, the $\rho$-values we give as examples will therefore represent the z-score rather than the raw srore.

\footnotetext{
'This assumption represents only an approximation since words in English are definitely not independent, but are dis. tributed according to the rules of the language.

"This notion is related to that of mutual information [4].
} 
Table 2 compares the list of LAs for the mv manual page ranked by frequency and by resolving power. In it. the LA (file move) has a greater resolving power than any of the following LAs. Moreover. some noisy LAs such as (do file) or (be file) (in italic fonts in the table) have disappeared because both words involved in the LAs are highly frequent in the corpus and thus have a low quantity of information.

\begin{tabular}{|l|l|}
\hline LAs & freq \\
\hline file move & 9 \\
be file & 8 \\
directory file & 7 \\
file system & 5 \\
file overwrite & 5 \\
file mv & 5 \\
file name & 4 \\
name path & 3 \\
do file & 3 \\
directory move & 3 \\
\hline
\end{tabular}

\begin{tabular}{|l|l|}
\hline LAs & $\rho$ \\
\hline file move & 8.38 \\
file mv & 4.36 \\
directory file & 4.03 \\
file overwrite & 3.87 \\
directory move & 1.98 \\
file system & 1.95 \\
mv rename & 1.71 \\
move mv & 1.58 \\
different file & 1.40 \\
name path & 1.33 \\
\hline
\end{tabular}

Table 2: Comparison of frequency and $\rho$-value for the LAs in $\mathrm{mv}$

For each document, we select as indices those LAs with the highest resolving power. More precisely, we are interested in the LAs that represent peaks in the distribution of $\rho$-valnes. Therefore, we keep as indices only the LAs whose $\rho$ value is one standard deviation above the mean, i.e., such that $\rho \geq \bar{\rho}+\sigma$, where $\bar{\rho}$ represents the mean and $\sigma$ the standard deviation of the distribution of $\rho$ values within one document. The choice of such a threshold ${ }^{6}$ is reflected in Tables 2, 3 and 4 , where only LAs with a z-score greater than 1 are presented.

The set of LAs of a document selected by ranking $\rho$-values and taking those one standard deviation above the mean forms the signature of the document. The major contribution of this technique consisted in adapting the notion of lexical affinity for indexing purposes. We gave some intuitive indications on how an $L A$-based indexing scheme is richer than a single-word scheme. We will demonstrate later that it ensures a better retrieval effectiveness.

The next section explains how software components can be stored and classified using the signatures produced at the indexing stage.

\section{The Classifying Stage}

Normally, when a user wants to use a software library, s/he first has to arress a library that might contain the desired component, then has to provide a formal description of the researched component according to the vocabulary understood by the library system. Unfortunately, in most cases, this ideal scenario does not work ont. The main reason is that in real life applications, the component perfectly matching the user's requirements does not exist in the library, or it is not indexed as the user had guessed it would be.

In such cases, a traditional database management system fails to help the user. Indeed, to be retrieved from the database. a component must exactly match the query ${ }^{7}$. Such strict matching is inappropriate

\footnotetext{
${ }^{B}$ This classical threshold grarantees to keep only a small percentage ol the sample elements in most distributions.

${ }^{7} \mathrm{~A}$ notable exception is ARES [18], a relational databse that allows flexible interpretation of queries. In ARES the
} 


\begin{tabular}{|l|l|}
\hline LAs & $\rho_{z}$ \\
\hline copy file & 6.49 \\
directory file & 2.47 \\
file source & 2.15 \\
infile subdirectory & 1.98 \\
contain subdirectory & 1.30 \\
copy cD & 1.30 \\
copy regular & 1.02 \\
\hline
\end{tabular}

Table 3: LAs ranked by $\rho$-values for $\mathrm{cp}$

\begin{tabular}{|l|l|}
\hline LAs & $\rho$ \\
\hline directory make & 5.08 \\
create mkdir & 2.74 \\
directory mkdir & 2.55 \\
directory permission & 1.48 \\
directory write & 1.03 \\
\hline
\end{tabular}

Table 4: LAs ranked by $\rho$-values for mkdir

in a software library system since the user often cannot know the exact characteristics of the desirable component and, even when $s /$ he does, there is rarely a perfect match.

Software libraries should not only permit retrieving candidate components that perfectly or partially match the query, but also permit browsing among components that share some functionality. It is therefore desirable to structure the library for making the search, retrieval and browsing mechanisms as fast, and convenient as possible, in order to make the access to the library attractive.

We propose here to perform the search and retrieval operations using a conventional inverted index file structure, and to ruster the library in order to facilitate the browsing operation. Section 4.1 explains how the index repository is built using an inverted file structure, and Section 4.2 presents the clustering technique used to build the browse hierarchy. Section 5 explains how they are nsed to perform the search and browsing operations.

\subsection{Building the index repository}

The goal is to allow a fast and easy identification of candidate components at the retrieval stage. Thus, we derive from the signature repository built at the indexing stage another repository for storing, for each word, the LAs involving that word, and pointers to the documents in which it appears. Let is denote:

- $W$ the universe of words

- $\mathcal{D}$ the universe of documents.

Index LAs are defined as tuples $\left(w, w^{\prime}, \rho\right)$ where $w$ is smaller than $w^{\prime}$ in the lexicographic order and $\rho$ is the resolving power of this LA in a considered docnment. The reason for ordering $u$ and $w^{\prime}$ is to avoid

similarity between elements can be evaluated via a lookup in a table that has to be provided beforehand. ARES is not discussed here since its purpose is not to classify software. Further, it has the drawback of requiring a great deal of preencoded knowledge. 
duplicate LAs by forcing every LA into a canonical form.

The index stored in the reposilory is represented as a mapping defined as follows:

$$
w \in \mathcal{W} \rightarrow \lambda(w)=\{(x, \rho, d) \in \mathcal{W} \times[1, \infty[\times \mathcal{D} \mid \text { either }(w, x, \rho) \text { or }(x, w, \rho) \text { is an LA of } d\}
$$

The mapping $\lambda$ is stored as a trie data structure. The mapping $\sigma$ between documents to their signatures is also stored using a trie data structure:

$$
d \in \mathcal{D} \rightarrow \sigma(d)=\left\{\left(w, w^{\prime}, \rho\right) \in \mathcal{W}^{2} \times\left[1, \infty\left[\mid\left(w, w^{\prime}, \rho\right)\right\} \text { is an LA of } d\right\}\right.
$$

In implementing these mappings, tries are usually faster than hashing schemes, although they consume more memory. In this case, fast access is a basic requirement for making the retrieval stage attractive. These two mappings are the basic operations we use to retrieve and rank candidates as explained in Section 5 .

\subsection{Building the browse hierarchy}

As explained previonsly, browsing is crucial in software library systems. The most common way to make browsing operations possible is to group items judged to be similar by using clustering operations [31]. Jardine and van Rijsbergen [19] pointed out that "associations between documents convey information about the relevance of documents to requests". They demonstrated that cluster-based retrieval strategies are as effective as linear strategies and much more efficient. Thus, many clustering methods have been used for information retrieval [19], (7], [16]. The most popular clustering methords are the hierarchical agglomerative clustering (HAC) methods because their search and construction techniques are more efficient than for most non-hierarchical methods [19].

The following sections define some terminology in cluster analysis, describe the algorithms we used to build the browse hierarchy, and present some samples of the browsing hierarchy obtained for the AIx library.

\subsubsection{Some terminology in cluster analysis}

Classification by clnster analysis has been of long-standing interest in statistics as well as various other fields. It can be traced back to the work of Adanson in 1757 [1], who used numerical clustering for classifying botanic species. Statisticians and taxonomists have widely developed the field since then. Cluster analysis now offers a wide range of techniques for identifying underlying structures in large sets of objects and revealing links between objects or classes of objects. One partirular application of classification is the building of libraries.

There is no strict definition of cluster, but it is generally agreed that, a cluster is a gronp of objects whose members are more similar to each other than to the members of any other group. Typically. the goal of cluster analysis is to determine a set of clusters, or a clustering, such that inter-cluster similarity is low and intra-cluster similarity is high. The similarity between objects is evaluated via a numerical measure called a dissimilarity inder defined as follows.

Definition 1 let $\Omega$ be a set of objects. A dissimilarity index $\delta$ over $\Omega^{2}$ is a function from $\Omega \times \Omega$ to $R_{+}$ that satisfies the following properties,

(i) $\forall 0 \in \Omega, \delta(0,0)=0$, 
Note that a distance is a dissimilarity index but that a dissimilarity inder does nnt necessarily satisfy the tringle inequality and therefore is not a distance.

The dissimilarity index between objects is used as the basic criterion to determine clusters. Clustering techniques allow identifying not only clusters but also relationships among them. The structure of the set of clusters as well as their internal structure vary with the clustering technique. Clustering methods are usually classified ${ }^{8}$ according to the structure of the set of clusters produced, e.g, hierarchical, flat. overlapping. etc., as well as the technique used, e.g. divisive, agglomerative. incremental, etc. As explained previonsly, hierarchical agglomerative techniques are very convenient for building browse hierarchies. The basic principle that these techniques follow is presented below.

Hierarchical numerical clustering aims at building hierarchies, over a set of objects. in which each internal node corresponds to a cluster of objects and each leaf represents an individual object, or more precisely a singleton cluster. Most hierarchical clustering methods are based upon the same general method. called the Hierarchical Agglomerative Clnstering (HAC) method [12]. which consists of iteratively gathering objects into clusters. until only one cluster remains.

The HAC general method iteratively builds a sequence of partitions or lenfl clusterings of $\Omega$. that is, a sequence of disjoint clusters covering the original set of objects. $\Omega$. The level clusterings form coarser and coarser partitions by an iterative process, beginning with the level clustering formed by the set of singletons in the power set $p(\Omega)$, i.e., $\left\{\left\{o_{1}\right\},\left\{o_{2}\right\}, \ldots,\left\{o_{n}\right\}\right\}$. and ending $" p$ with the coarsest partition of $\Omega$. i.e., $\{\Omega\}$. The final output of this clustering process is a particular form of hierarchy called a dendogram. The HAC general method can be expressed as follows:

- Start with the subset of $p(\Omega)$ formed by singleton elements.

- Repeat the following steps iteratively until there is only one cluster.

- Identify the two clisters that are the most similar.

- Merge them together into a single cluster.

The IIAC method reqnires a measure of similarity not only over the set of objects, but also over the set of clusters. The dissimilarity index between clusters is usually derived from a user-given dissimilarity index. $\delta$, belween objects. The way of defining $\Delta$ has a direct influence on the final form of the hierarchy obtained. Once a dissimilarity index $\delta$ between objects is provided. HAC mnthods differ only by the choice of this measure. The most commonly used HAC methods are the single link and complete link methods [22]. Many other methods such as the centroid method. Ward's method, otr., infine still other dissimilarity indices but most of them require the dissimilarity index over $\Omega$ to he a listance, that is, to satisfy the triangle inequality. The reader should consult [13] [12] for an extensive survey of the HAC methods. The time complexily of the HAC algorithm is at most $O\left(n^{2} \log n\right)$ where $n$ is the number of ohjects involved. For some particular definitions of $\Delta$, it can be reduced to $O\left(n^{2}\right)$.

"With the recent introduction of conceptual clustering [29], another distinction has heen introduced according to the definition of the clusters obtained, in extension (i... by enumeration of its members) for regular (or numerical) clustering and in intension (i.e., by membership rules) as well as in extension for conceptual clustering. 


\subsubsection{Adapting a clustering technique for building a browse hierarchy}

As explained above, we propose to use a $\mathrm{HAC}$ technique to generate a browse hierarchy. In this perspective. we (1) need to define a measure of similarity between the objects considered. e.g., the documents. and (2) explain how to make a browse hierarchy ont of the dendogram generated by the HAC technique. Let ns address these two points.

In information retrieval, numerons measures of similarity between documents, also termed measures of association or coefficients of association, have been defined. The simplest of all is defined as:

$$
|X \cap Y|
$$

where $X$ and $Y$ are the signatures of two documents. This measure represents the number of common index units. Varions other measures [38] have been defined such as:

$$
\begin{array}{ll}
\frac{2|X \cap Y|}{|X|+\left|Y^{\prime}\right|} & \text { Dice's coefficient } \\
\frac{|X \cap Y|}{\left|X \cup Y^{\prime}\right|} & \text { Jaccard 's coefficient } \\
\frac{|X \cap Y|}{|X| \times\left|Y^{\prime}\right|} & \text { Salton's Cosine coefficient }
\end{array}
$$

They can all be considered as normalized versions of (9) since they are functions of the cardinality of $X, Y, X \cap Y$, or $X \cup Y$.

In onr context. we have more information than just the presence or absence of index $u$ nits in the signature, and therefore we propose to take into account the $p$-values of LAs in the evaluation of the measure of association between documents. For any signature $X=\left\{\left(w, w^{\prime}, \rho\right)\right\}, p(X)$ is the projection set of $X$ over $\mathcal{W}^{2}$. Then, the simplest measure is $|p(X) \cap p(Y)|$. In order to take into account the resolving power of LAs as well, we define our measure $\delta$ for two signatures $X$ and $Y$, such that $X \neq Y$, as

$$
\delta(X, Y)=\sum_{\left(w, w^{\prime}\right) \in p(X) \cap_{p}(Y)}\left(\rho_{X}\left(w, w^{\prime}\right)+\rho_{Y}\left(w, w^{\prime}\right)\right)
$$

where $\rho_{x}\left(u^{\prime}, w^{\prime}\right)$ is the $\rho$ value of the LA $\left(u, w^{\prime}\right)$ in the signature $X$. and similarly for $Y$. Note that $\delta$ is a measure of similarity rather than a measure of dissimilarity. Its inverse is a measure of dissimilarity as long as $\delta(X, X)$ is set to a sufficiently large arbitrary value so that its inverse can be considered essentially null.

Given such a measure of similarity between signatures, we define a measure of similarity between clusters according to the single link or complete link techniques for instance and then use the hierarchical agglomerative clustering algorithm in order to build a browse hierarchy of snftware components. Let. us note that we also made some experiments in earlier versions of Gunu using an incremental conceptual clustering technique [25] for constructing the browse hierarchy. However, reapite interesting results, the cost of building and maintaining the hierarchy was prohibitive (exponential time like for most conceptual clustering techniques) when compared to regular clustering techniques and did not appear to be better in terms of retrieval effectiveness.

All the HAC techniques build a binary hierarchy. Not all levels of the hicrarchy are equally significant; therefore, the usual approach is to select manually the most significant level clusterings, this task being usually performed by a data analyst. The following proposes a method for allomatically identifying the most useful level clusterings, and thus producing a not-necessarily binary hierarchy.

This method of selection is based on the following principle. Each level clustering in the dendogram corresponds to the merging of two clusters in the previons level clustering and therefore to a particular 


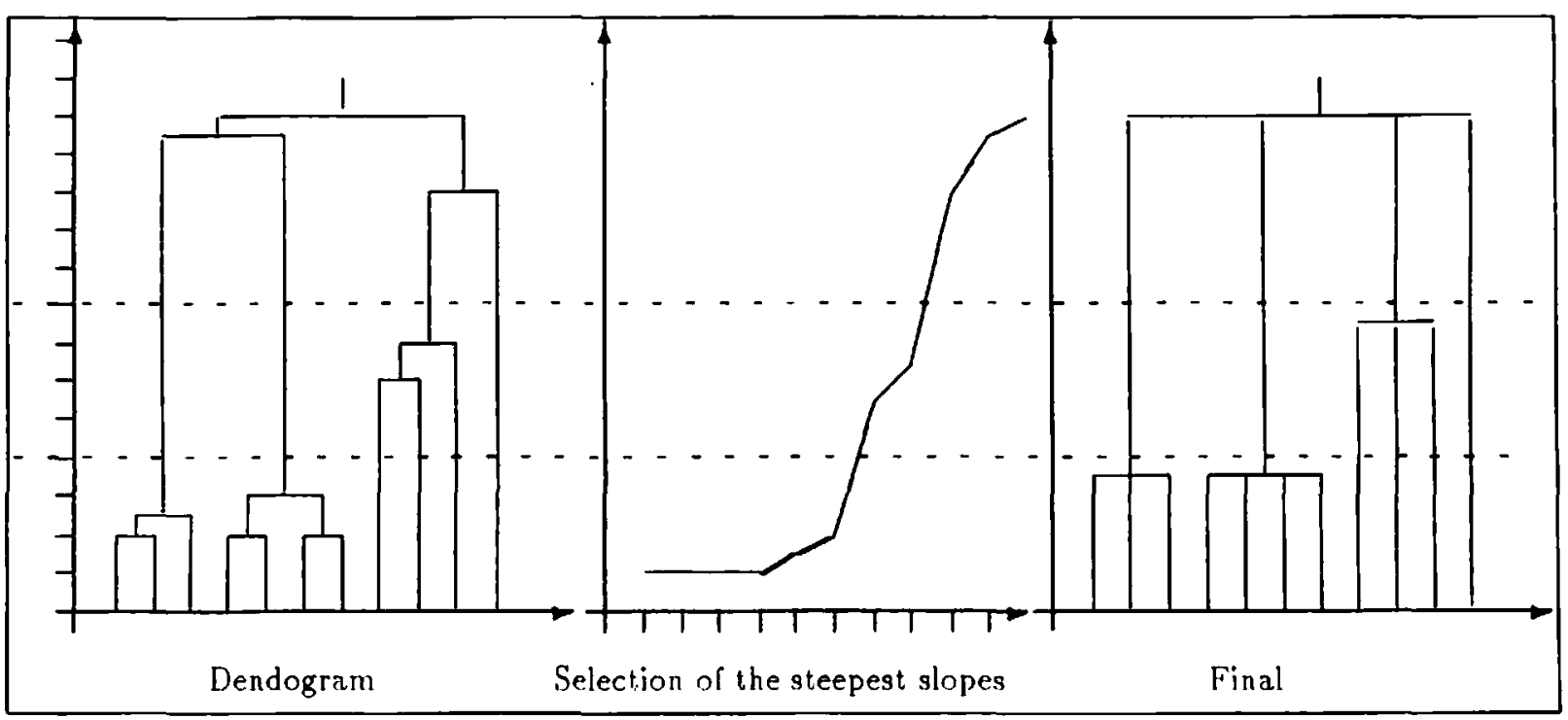

Figure 2: Principle of selection of level clusterings

value of the similarity measure. If we label the dendogram with these values $y_{n}, \ldots, y_{1}, n$ being the number of objects, from the bottom to the top of the hierarchy, it can easily be shown that the $y_{i}$ 's are (non-st,rictly) monotonic (increasing for dissimilarity measures and decreasing for similarity measures) for the single and complete link clustering methods. We propose to select those levels that correspond to the gap in the distribution of $y_{i}$ 's by (1) plotting the segment connecting the pairs $y_{i+1}, y_{i}$ from $i=n-1$ to $i=1$, and (2) keeping the levels that correspond to the steepest slopes. This represents the intuitive method that a data analyst would apply. Figure 2 gives an intuitive presentation of the method via an example whereas Figure 3 gives the formal algorithm. The time complexity of the latler is linear in the number of objects.

\subsection{Some examples}

Portions of the browse hierarchy built from the Alx documentation are shown in Fignres 4 and 5 . In Figure 4. some interesting clusters are isolated. Thus, in the figure we have a cluster gathering commands related to the manipulation of regular expressions, and a cluster gathering editors. These two clusters are also part of the same super-cluster, mainly because these editors permit to manipulate regular expressions. Then, there are two ontliers that could not be included in a cluster: makekey and termdef. Then a small cluster groups ps and kill, which both are strongly related as they give information about processes or handle them. Finally, there are two big clusters, one for yellow pages commands and another for SCCS routines. The clustering is not always of such good quality as can be seen in Figure 5, either because of the nature of the documentation or because of the principle of clustering itself. For instance, the commands $x c a l c$ and dc. which both are calculators. belong to a same cluster, but be has been forgotten in this cluster. This is due to the fact that the manual page of be does not refer to the concept of calculator at all, but defines bc as an interpreter for an arithmetic language. The real problem with clustering is illustrated with the third cluster in this figure, which gathers batch, at. crontab, date and istat. This cluster has been formed because all these commands are related to the notion of date or time; unfortunately, this is not the main functionality of all of these commands and therefore this cluster is somehow misleading. Let 


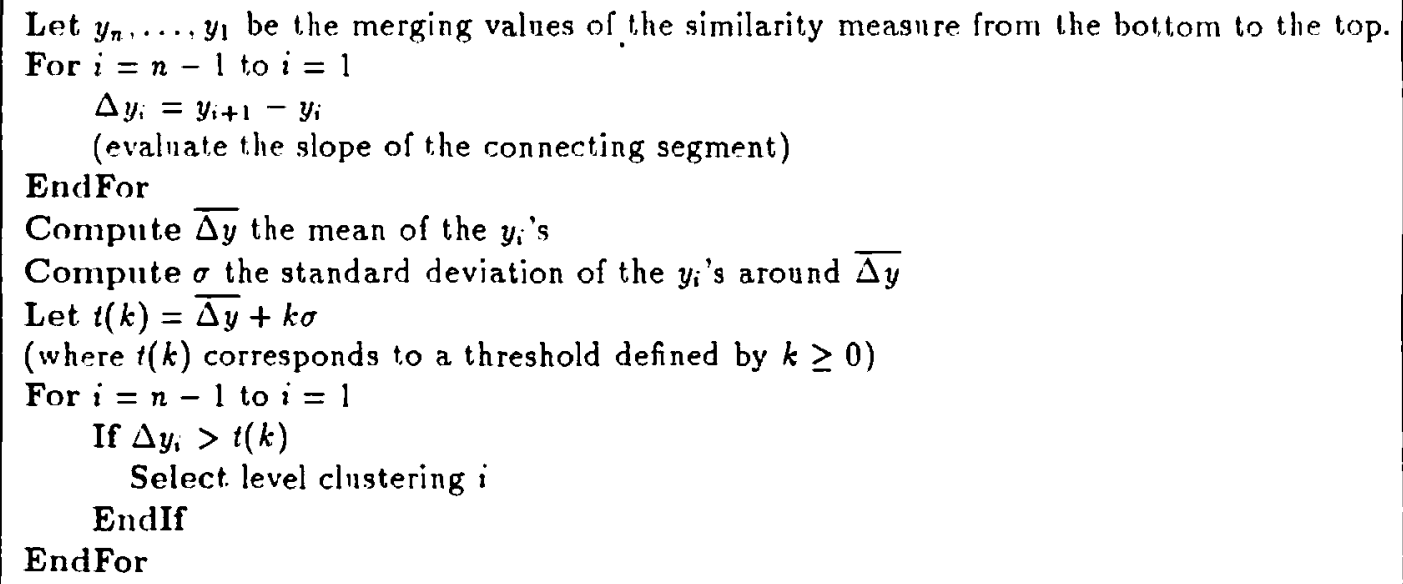

Figure 3: Selection of level clusterings

Is note. however, that the lower level cluster including at and batch is a good one.

The hierarchy thus generated is used as an aid to browse when nothing relevant has been retrieved via linear retrieval, or in order to increase recall since there is no way to be sure that all the relevant components have been retrieved at the linear retrieval stage. It can also be 1 sed as the basic repository to be searched during retrieval, but we prefer to use the traditional linear retrieval technique instead because it is clearly more trustable considering the problems described above.

By nature this indexing technique suffers from noise since it is based on only statistical observations. Noisy indices involve generally misspelled or unmeaningful strings of characters that are mixer with natural language (for describing instructions for instance), or "side-concepts" such as the time, day and month in the example rited ahove. This noise cannot be avoided when dealing with free-style text.

Fortunately, these noisy $L A$ s do not cause real trouble at the linear retrieval stage since there is a very low probability that the user would use unmeaningful character strings in her/his queries. So noisy LAs are part of the signatures of components but rarely lead to the selection of the considered component. On the other hand, noisy LAs might induce the formation of poor quality clusters, but generally only higher levels of the hierarchy are affected since "side concepts" are not given much weight when evaluating similarity. Section 5.3 explains how this browsing hierarchy is used at retrieval stage.

\section{The Retrieval Stage}

The previous sections explain how libraries of reusable components are assembled. We also need to be able to retrieve the components that match the requirements when at least one exists, or to assist in the selection of the closest components via a browsing facility.

The usual scenario when retrieving a component is the following:

- Query specification: The user expresses a query according to the anthorized vocabulary. 


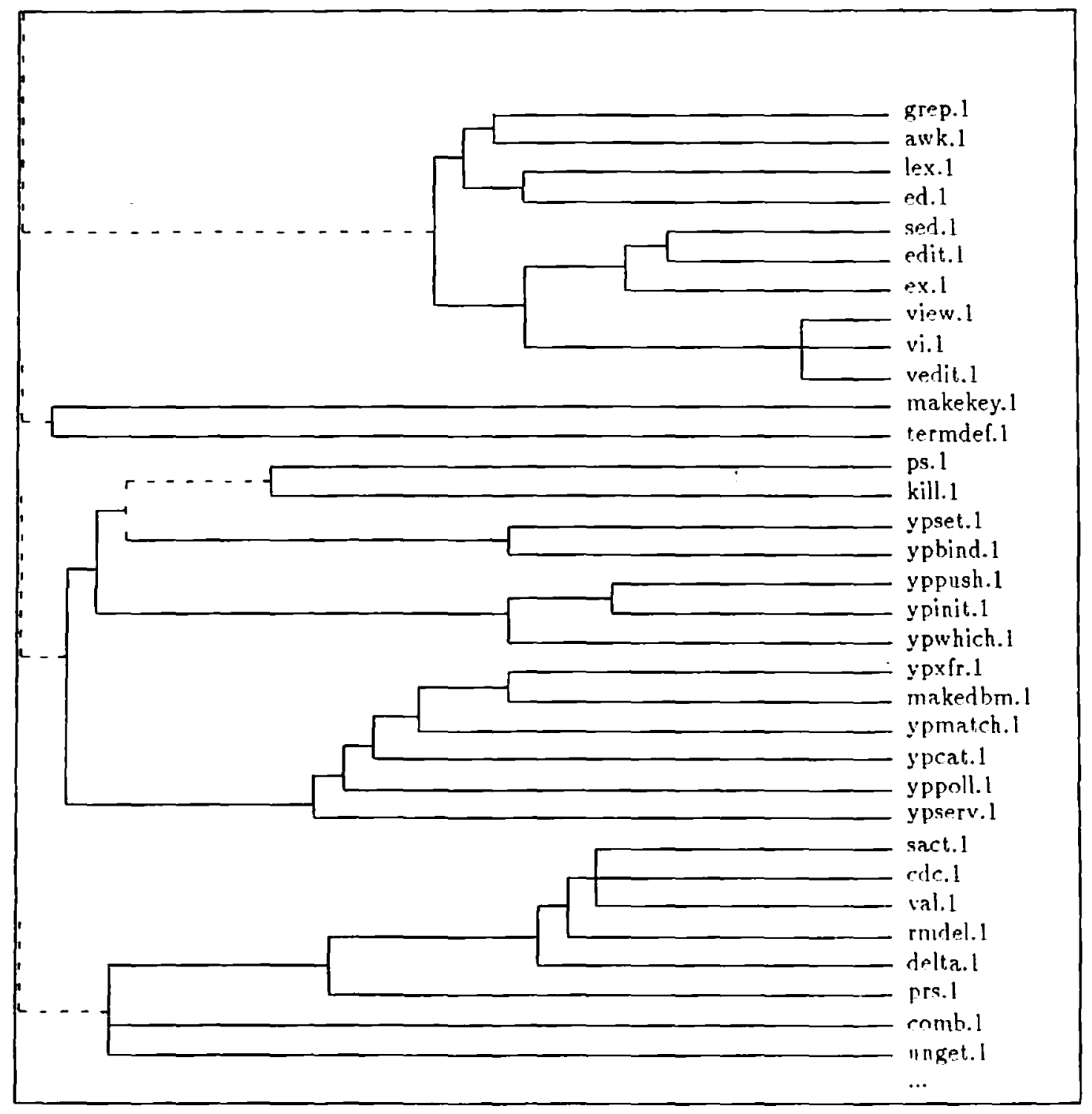

Figure 4: Portion of $\Lambda \mathrm{IX}$ hierarchy (single link. $k=0.5$ ) 


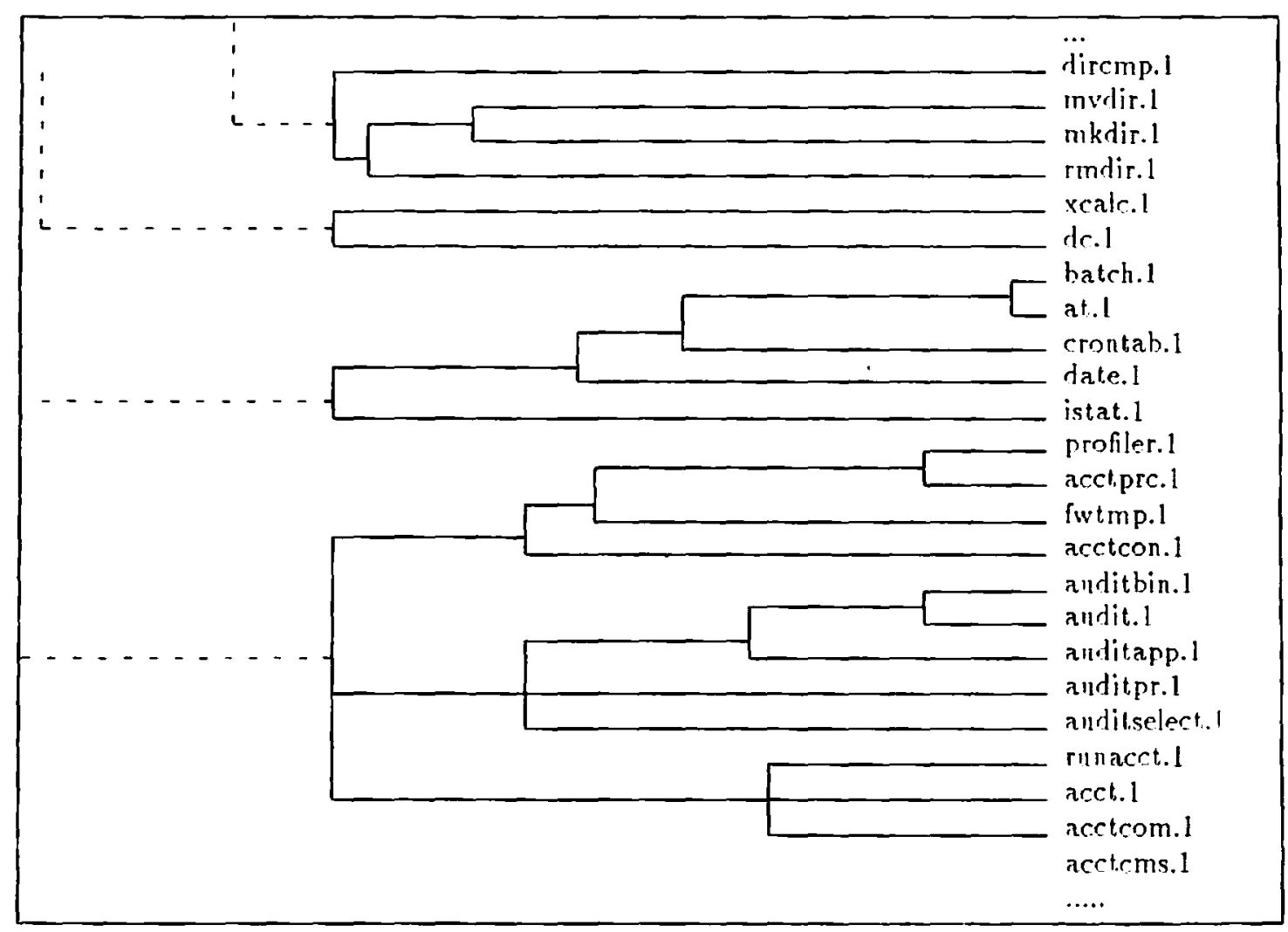

Figure 5: Portion of A $x$ hierarchy (single link, $k=0.5$ ) 
- Linear retrieval: A search locates the candidate components and the candidates are ranked arcording to their degree of match with the guery.

- Browsing Cluster-based retrieval is initiated when no adequate components have been found by the linear retrieval.

The following explains how these three stages are supported in our approach.

\subsection{Query specification}

Using uncontrolled-vocabulary indexing as we do presents clear advantages at the query specification stage. Indeed, a minimum of constraint is put on the user as $s /$ he expresses her/his query. The nser does not have to learn a specific index language or understand the organization of the library. S/he can express her/his query in natural language and then the indexing component is applied in order to t,ranslate the query in to attributes understandalue by the system. Exactly the same terhnique is used for extracting LAs from natural-language queries as from natural-language documentation. This provides a very convenient and user-friendly interface between the user and the library system, beralse the user is not constrained by any rigid formalism.

The queries can be expressed in free-style natural language. However, the user must be aware of the fact that queries are not really interpreted. but rather considered as a description of the functionality of the desired component. For instance, the nser could express queries of the form "How can I do such and such" since only the "such and such" would be considered for indexing. the rest, heing either closed-class words or words with low quantity of information. Formulating a query that necessitates some understanding. such as a query including negations like "but not.". wonld only lead to wrong interpretation. Let us note that it wonld be possible at this point to allow some simple interpretation of the queries. by allowing for instance the usual boolean connectors ("and". "or". "but not"). This would clearly boost the performance of the library system. However, since our point here is to show how far we ran go without understanding either the gueries or the documents. we do not discuss these possible anliancements.

\subsection{Linear retrieval}

In order to retrieve the hest candidates for a given query, we apply the usual IR method, which consists of considering the query as a docunent and retrieving the components in the repository whose signature are the most similar to the signature of the query. A possible measure of similarity is the $\delta$ measure defined in (13, Section 4.2.2. The most similar components are then relurmint to the user. ranked in order of decreasing similarity with the query. The linear retrieval technique is promentod in Figure 6.

In case of low recall, that is, if the user is not satisfied with the retrimurel randidates, a more fuzzy search can be performed that also considers partial matching LAs. In that rase only $I, A$ s that partially match a query $L A$, i.e. have one word in common, are considered. This signifirantly increases the recall but as a tradeoff drastically decreases the precision. It shonld therefore he usod only when the user considers that nothing relevant has been retrieved with the initial query. An example of linear retrieval is given in Figure 7.

In Figure 7 , the candidates are ranked in order of decreasing similarity with the query ("How can I locate regular expressions in a file"). Therefore, the top candidates nsually answer the query the best. In the example shown in Figure 7 , all the candidates retrieved deal more or less strongly with regular 
Get natural-language query from user

Index query and produce its signature $Q=\left\{\left(u, u^{\prime}, \rho\right)\right\}$

For each query $\mathrm{L} A,\left(w, w^{\prime}, \rho\right) \in Q$

$C\left(w, u^{\prime}\right)-\left\{c \mid \exists \rho\right.$, such that $\left.\left(w^{\prime}, \rho, c\right) \in \lambda(w)\right\}$

(i.e., identify all the components that have this $I A$ in their signature)

EndFor

$C-\cup\left\{C\left(w, w^{\prime}\right)\right\}_{\left(w, w^{\prime}, \rho\right) \in Q}$

For each $c$ in $C$

Evaluate the similarity between the signature $S_{c}$ of $c$ and $Q$ as $\delta\left(Q, S_{c}\right)$

(where $\delta$ is the similarity measure defined in (13))

Rank components in order of decreasing similarity.

Figure 6: Linear retrieval technique

Proressing query:

How can I locate a regular expression in a file

Lemmatizing sentence...

Searching...

regex.3 220.21

regexp.3 220.21

awk.l $\quad 7.32$

grep.l $\quad \overline{7} .32$

find.1 33.88

ogrep.l 28.77

regcmp.3 28.75

dosfirst..3 22.38

dosnext.322.38

Figure 7: Example of linear retrieval 
Processing query:

Establish a new password.

Lemmatizing sentenre...

Searching...

passwd.l 32.05

Linear retrieval

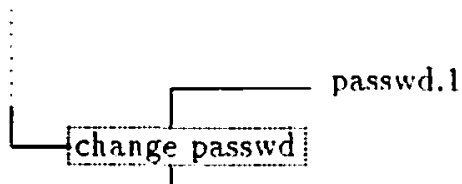

yppasswd.1

Cinster-based retrieval

Figure 8: Browsing in the hierarchy

expressions. Even the l,wo last candidates. dosfirst and dosnext, do not, answer the query, but are very slightly related since they allow locating DOS files that match a patitern.

\subsection{Browsing, cluster-based retrieval}

The retrieval stage in classical library management systems is often limited to locating a set. of components exactly matching the user's query or, when such components do not exist, related components. Library systems do not. usually provide any further assistance, whereas many IR systems do.

In our approach, the user may communicate interactively with the system in order to direct the browsing when $s /$ he is not satisfied with the first retrieval yielded. The linear search retrieves the most related randidates, and then the browsing process begins.

Typirally, the user starts from one of the candidates retriever by the linear search and explores the hierarchy bottom-up. Consider the browse hierarchy given in Figure 4 and suppose that a user gives a query asking about ways "to identify a process". If the first candidate retrieved at the retrieval search is $k i 11$. Then, the user can access the browse hierarchy, and explore the clusters including kill in order to determine which components are strongly related. In onr example, s/he will find ps as the most related component, which is clearly a better candidate for this given query than the one retrieved by the linear soarch. Ancthar rxample is illustrated in Figure 8. The two relevant candidates in Aix for the query "estahlish a new password" are passod and yppassud. However, the linear retrieval retrieves only passud simply because the query had no intersection with the signature of yppassud. At this point. the user could reformulate the query, but s/he might not be aware that s/he has missod sone relevallt candidates. Using the browse hierarchy is therefore more ennvenient in order to rherk if some unexperterl candidates have been missed. In the example, both pasoud and yppassud are stirongly relatirl: their signatines share the LA (change passud) Browsing in the hierarchy from passod allows the nser to retrieve the other relevant candidate. These two examples show how a browse hierarchy can help improve the finding of possible candidates that conld be missed via linear retrieval.

At any point, the user can consult the signature of a component in order to have more information about its functionality. Fast access to signatures is achieved via the signalure repository. The user can also provide, at any stage, fnrther information in order to get a finer retrieval. By browsing, s/he gets

\footnotetext{
"Note that "passwd" here is proper name and is different from the noun "pasavord" mentioned in the query.
} 
more information about components and learns how to provide discriminating queries.

\section{Empirical Results}

The approach described in the previous sections has been embodied in a tool. Gunu, whirh has been fully implemented, partly in VSPascal and partly in (. under AIx. The system lias reached a satisfactory first stage, and the implemented version yields quality results.

We have tested our system on the entire $A t x$ documentation available to $n s$, which describes approximately $1,100 \mathrm{~A} I \mathrm{x}$ components. When building the index repository, we therefore processed the entire documentation that forms a corpus of more than 800,000 words, and we identified $18,000 \mathrm{I}$, As for the 1,100 signatures.

In order to evalnate Guru's performance, we nsed the following criteria.

- User effort. This consists of all the effort that must be expended by the user in order to use the library system. It is impossible to formally measure user effnrt. However, thanks to the uncontrolled vocabulary approach that we applied, we believe that the effort that must be invested for using GURU is minimal. Queries can be formulated in natural language, ant therefore the user is not required to learn any index language and formalism.

- Maintenance effort. This consists of all the effort that is neressary to keep the system working and up to date. This effort, includes, in particular, indexing new components and adding them to the library. The maintenance stage is highly facilitated in GuRu. The indexing is performed automatically and the insertion of new components can be done incrementally. Kaplan and Maarek. in [20], have proposed several algorithms for incrementally updating a rapository of 1 A-based indices when inserting, deleting or modifying components.

- Efficiency. This refers to the average interval between the time a query is issued and the time an answer is given. Efficiency becomes an issue only if a rel,rieval takes so long that, users start to romplain. Onr experience with the system shows that efficiency is not an issue, as the response time is reasonable. Profiling the execution of the query prngram showed that the time to perform the query was dominated by the time to map the repository file into the address space of the query program. The lookup operations and the printing of the LA-file name pairs consumed almost no time in comparison. Test queries involving from 5 to $15 \mathrm{~L}$. As earl tonk approximately 2.5 seconds on an RT, and 0.15 seconds on an IBM RISC System/f0n0. The helter performance of the latter is partly due to its more efficient implementation of file mapping.

- Retrieval effectiveness. This is rearly the most important performalle rriterion. It refers to the system's ability to provide information servires as needed by the us $r$ r.

The next section focuses on evaluating the retrieval effectiveness of (iupu. 


\subsection{Measuring Retrieval Effectiveness}

\subsubsection{Recall and Precision}

The most widely used measnres for evaluating retrieval effectiveness are recall and precisinn [32]. Recall is defined as the proportion of relevant material, i.e.. it measures how well the considered system retrieves all the relevant components. Precision is defined as the proportion of retriever material that, is relevant. i.e.. it measures how well the system retrieves only the relevant compononts. Recall can also be interpreted as the probability that a relevant component will be retrieved, and precision as the probahility that a retrieved component will be relevant [5].

Recall and precision can be defined more formally as follows. I, et $C$ be the whole collection of components forming the library. For each query. $C$ can be partitioned into two disjoint sets. $R$, the sel of relevant material and $\bar{R}$ the set of irrelevant material. Given the query, the system retrieves a set of components $c$ that can also be partitioned into relevant and irrelevant material, respectively, $r$ and $\bar{r}$. Recall and precision are defined as:

$$
\begin{aligned}
\text { recall } & =\frac{r}{R} \\
\text { precision } & =\frac{r}{c}
\end{aligned}
$$

Recall and precision measurements require the ability to distinguish between relevant and irrelevant material. For relatively small collections such as the AIX collection, it is possible to manually determine the set of relevant material for a given query.

\subsubsection{Experiments and Comparison}

This section describes the experiments that allowed us to evaluate the retrieval effectiveness of GuRU. As a hasis for comparison, we have considered INFOExFLORER, which is an IBM RISC: System/6000 CD-Rom Hypert,ext. Information Rase I,ibrary. INFOF,XPLORER is a recent, hypertext. system that gives access to the documentation for AIX and for associated programs. InFoFixpionfon provides not only liypertext links between pieces of the AIx documentation, but also search and retrieval farilities based on state-of-the-art. IR terhniques. Queries ran be expressed as single word search nr multiple word compound search with no control of vorabulary. The compound scarch, which is the most elahnrated, allows the uscr to express a query as a word pattern formed of single words related by thrm pnssihlo rnnnertors, "and". "or". and "but not". Moreover, the user can restrict the search. S/he can giw" innstraints specilying if the pattern words must appear within the same articte or within the same paragraph, the proximity of these words within a paragraph, and the search fields and the search rategorins.

When given such a query. INFOExploner. relurns a list of randidales that exactly fit the query, ranked according to the frequency of the pattern in the considrerd dorument. No signature is built for the documents examined: all words appearing in the text are ennsirlerert during search. Therefore. INFOEXFICnFR. can be expected to have a a much higher recall but lower precision than GuRU. We do not need to also compare efficiency, i.e., retrieval speed. Gurul is, independently of implementation, much faster than INFOExpLORER since it does not explore the entire textual database but, a much smaller repository formed by the signatures.

INFOEXPLORER is thus a quite sophisticated IR tool that represents a good reference lor comparison purposes since it is specifically for AIX. Also. INFOEXPLORER encodes a great, deal of manually-provided 
information about the structure of the documentation. The system has lon know about. paragraphs, litles, etr., and thus has been much more expensive to build than Guru. Proviling this structural information to onr system wonld greatly enhance its performance, but our point here is to show that even wilhout such information, our system can perform nicely thanks to its indexing srheme.

GunU and INFOEXPIORFR were compared for retrieval effectiveness. In order to claim this test to be valid, we must fulfill the usual test, procedure requirements [32]. These roquirements are for

1. the queries to be used for test purposes must be user search requests actually submit,ted and processed by hoth systems:

2. the test collection must consist of documents originally inclided in the library, chosen in such a way that any advance knowledge concerning the retrjevability of any given component by ejther system is effectively ignored; and

3. the number of components considered to be retrieverl by the twn systems must be subject to the same cutoff.

To fulfill the first requirement, we have conducted a survey among the graduate students in the Department of Computer Science at Columbia University in November 1088. "This survey provicled us with a collection of typical queries on INIX-like systems, as formulated by ['NIX users ranging from naive users to expert programmers. $\Lambda$ typical query was expressed as a naturalilanguage sentence with an average of 3.7 open class words per query, describing a desired functionality. This kind of query could direclly be fed to GURU but not to INFOEXFLOREn since the latter's compound search facility arcepts only boclean queries. Therefore, foeding the queries to INFOEXPLORER required some supplementary effort, first choosing the right connectors between open-class words extracted from the queries, and possibly dropping some words when the recall was too low. In our interaction with the compoind search facility. we had to refine and retry the query formulation several times. We kept, only the best. result, for comparison purposes, since wo wanted tn compare the tools' indexing schemes rather than their querying facilities. Ciunu's querying facility requires less user effort than INFOEXPLONER's, but the lat ter's rould be greatly improved if it did not require perfect matrhes hetween the boolean query and the candidates, using a similarity measure hetween randidate and query, for instance. The average number of open-rlass words used for questioning INFOEXPIORFR was 3 .

As far as the second requirement is concerned, the collection considered for test has been the entire A IX lihrary. We consulted with several AIX experts at IBM in orfer to Hetermine for each query the set of existing relevant components in the AIX library sn as to be able to evaluate the recall and precision. As onr test collection was composed of about 1100 components, we selected 30 qurirs from among all the queries provided by nur survey. This ratio corresponds to the same number-of-rumeries per number-of-documents ratio as the one that has been used in standard test sets surh as Mrr. (rollertion of modical ahstracts, 30 queries for 1033 documents) or cisl ${ }^{10}$ (information scienen abstrarts, 35 queries for 14 fin information abstracts).

As far as the third requirement is concerned, since bot, systens ranked the retrieved candidates, we were able to compare recall and precision at the same ranks.

The romparison was performed by measuring. for both systems. prerisinn at, several levels of recall. We followed the usual procedure [38]. [32], which consists of

\footnotetext{
${ }^{10}$ These test sets have heen used for evaluating several IR systems such as ISSI [9]
} 
1. plotting precision-recall curves for each test query with each plot corresponding to a given culoff value,

2. extrapolating these curves so as to obtain precision values for recall values that were not effectively achieved, and finally,

3. deriving from the curves computed in stage (2) the average precision values at fixed recall intervals. so as to obtain a single average precision recall curve for the system considered.

We have built such curves for both GURIJ and INFOEXPLORER and plotted them on the same axes (See Figure 9). The hest performance is reached by the system whose curve is closest to the area where both precision and recall are maximized. the upper right corner of the graph. As mentioned, becalse of the indexing scheme of both systems, we could expect that INFOEXPLORER would achieve a lower precision but higher recall than (iunu. It turned ont that the maximum recall, all ranks included, achieved by both systems was approximately the same, around $88 \%$ on the average, but, from the graph presented in Figure 9. it is clear that Guru had $15 \%$, on the average, better precision than INFoExPLORER. In other words. Guny achieves a higher precision without losing in recall. This is more than satisfactory.

These results clearly prove that we have achieved high precision without losing recall. The recall rate is significantly increased when we make nse of the GuRu browsing facility. For instance, in several cases some related components were not, retrieved during linear retrieval, but, only during browsing.

The results of this evaluation should not be seen as the final definitive results, but only as an indicator of what can be expected from a fully operational GuRU system. However, even introspective experiments such as those described in this section are sufficient to confirm the advantages of an $\mathrm{L} A$-based indexing scheme. Our LA-based indexing scheme makes the indexing language exlianstive as well as specific and thus ensures good retrieval performance. The experimental results confirmed the expectations an can be considered as enconragements to pursue research in the direction pursued in this paper.

\section{Conclusion}

We have presented a method for automatically constructing software libraries from a collection of documented hut unindexed software components. We discussed the advantages of using natural-language documentation as opposed to source code, assuming any documentation is available, as a sonrce of functional information. We then described a new free-text indexing scheme, for antomatirally producing document signatures, based "pon a richer unit than single terms, the lexiral affinity. All associated software components could then be classified. stored. compared and retrievad, via litiear nr cluster-based terhniques. arcording to these indices.

These methods and schemes are embodied in a new tool which has hmen implemented and evaluated for retrieval effectiveness. The evaluation compared GuRU with the INFoFixroorfon hypertext library, built specifically to help find software components in the AIX system. The average recall-precision curves of both tools were computed. The results of this test indicate that. Culpul's performance was better than INFOEXPLORFR. This result is very enconraging since INFOEXRLORFR was murh more expensive to build and specifically tailored to the AIX library.

The major contribution of this work consists of bringing classical and new information retrieval techniques to bear in soltware reuse. This involved:

- Designing a new indexing scheme based on high information content. lexical affinities. 


\begin{tabular}{|l|l|l|l|}
\hline Recall & GuRU precision & INFo precision & Improvement \\
\hline \hline 0.1 & 0.85 & 0.7 & $15 \%$ \\
0.3 & 0.84 & 0.68 & $15 \%$ \\
0.5 & 0.76 & 0.56 & $20 \%$ \\
0.7 & 0.58 & 0.4 & $18 \%$ \\
0.9 & 0.52 & 0.39 & $13 \%$ \\
\hline
\end{tabular}

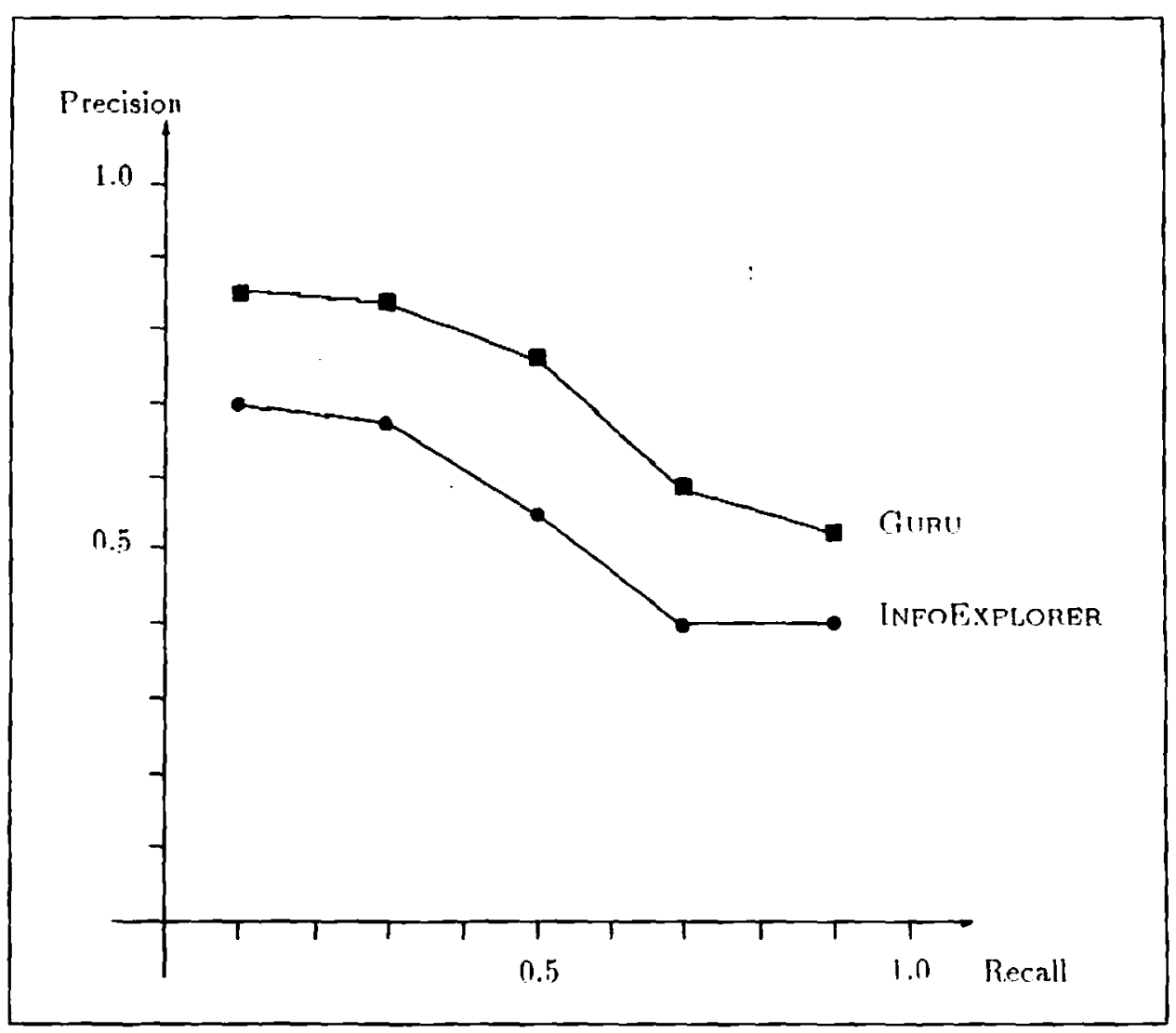

1

Fignre 9: Precision-recall curves (means across querina) 
- Arapting classical numerical cluster analysis techniques for assemhling software momponents into browse hierarchies.

- Designing retrieval mechanisms sperifically adapted to the LA-hased indexing scheme so as to provide a complete storage and retrieval framework.

Finally, the evaluation we have performed seems to indicate that, Salton's slatement about the limitation of the "phrase generation" approach in indexing (See Section 3.1) is overly pessimistic and that significant improvements over single terms techniques can be achieved at relatively low cost.

\section{Acknowledgments}

Y. Maarek performed part of this work while at, the Technion. Department of Computer Science, Haifa, Israel, partly supported by a Gutwirth Fellowship. G. Kaiser is supported by National Science Foundation grants (SA-8920080. CCR-8858029 and CCR-8802741, by grants from $\Lambda$ T.ET. BNR. Citicorp. DEC. IBM. Siemens. Sun and Xernx. by the Center for Advanced Technology and by the Center for Telecommunications Research.

We would like to thank Mark Kennedy who helped a lot in the design and implementation of Gunu's retrieval component.

\section{References}

[1] M. Adanson. Histoire Naturelle du Sénégal. Coquillages. Avec la relation abrégée d'un voyage fait en ce pays, pendant los années 1749.50,51.52 et 5.9. Bauche, Paris. France, 1757.

[2] B.P. Allen and S.D. Lee. A knowledge-based environment for the development of software parts composition systems. In Prnceedings of the $1 t^{\text {th }}$ ICSE, pages 104-112. Pittsburgh. PA. May 1989.

[3] S.P. Arnold and S.I. Stepoway. The reuse system: Cataloging anrl retrieval of rensable software. In W. Tracz, editor, Snftuare Reuse: Emerging Technolngy, pages 1.38-141. Computer Society Press, 1987.

[4] R. Ash. Information Theory. Interscience Publishers (John Wiley \&. Sons). New York. 1965.

[5] D.C. Blair and M.E. Maron. An evaluation of retrieval effertiveness for a full-text dornment retrieval system. Communications of the ACM, 28(3):289-299, March 1985.

[f] B.A. Burton, R. Wienk Aragon, S.A. Bailey, K.D. Koelher, and L.A. Mayes. The rensable software library. In W. Tracz, editor, Snftuare Reuse: Emerging Technningy, pages 129-137. Computer Society Press, 1987.

[7] F. Can and E.A. Ozkarahan. A clustering scheme. In Procerdings nf SICIR'83, pages 115-121. Bethesda, MID, June 1983. ACM Press.

[8] F. de Saussure. Cinurs de Linguistique Gènérale, Quatrième Editinn. Lihrairie Payot. Paris, France, 1949.

[9] S. Deerwester, S.T. Dumais, G.W. Furnas, T.K. Landaller, and R. Ilarshman. Indexing by latent semantic analysis. Journal nf the American Society for Information Srience, 11(6):391-407, 1990. 
[10] P. Devanbu. Re-11se of software knowledge: A progress report. In Third Annual Workshop: Methods and Tonls for Reuse. Syracuse. NY. June 1990.

[11] P. Devanbu, P.G. Selfridge. B.W. Ballard, and R.J. Brachman. A knowledge-based software in formation system. In Procedings of IJCAI'89. pages 110-115. Detroit, MI, Angust. 1989.

[12] E. Diday, J. Lemaire, and F. Testu. Eléments d'Analyse des Donnfes. Dunod, Paris. France. 1982.

[13] B. Everitt. Cluster Analysis. Halsted Press (John Wiley \& Sons), New York. 1980.

[14] W.B. Frakes and P.B. Gandel. Classification, storage and retrieval of reusable components. In N.J. Belkin and C.J. van Rijsbergen, editors. Proceedings of SIGIR 89. pages 251-254. Cambridge, MA, June 1989. ACM Press.

[15] W.B. Frakes and B.A. Nejmeh. Software reuse through information retrieval. In Proceedings of the 20th Annual HICSS, pages 530-535, Kona. HI, January 1987.

[16] A. Griffiths. L.A. Robinson, and P. Willett. Hierarchical agglomerative clustering methods for antomatic document classification. Journal nf Documentation, 40(3):175-205. September 1984.

[1i] W. Harrison. A program development environment for programming hy refinement and reuse. In Procedings of the 19th HICSS, pages 459-469. Kona. HI. 1986. CS Press.

[18] T. Ichikawa and M. Hirakawa. Ares: A relational database with the capability of performing flexible interpretation of queries. IEFE Transactions of Snftware Engineering. 12(5):624-634, May 1986.

[19] N. Jardine and C.J. van Rijsbergen. The use of hierarchic clustering in information retrieval. Information Storage and Retrieval, 7(5):217-240, December 1971.

[20] S.M. Kaplan and Y.S. Maarek. Incremental-maintenance of semantic links in dynamically changing hypertext systems. Interacting with Computers, December 1990. In press.

[21] P.H. Klingbiel. Machine-aided indexing of technical literature. Informatinn Starage and Retrieval. $9: 79-84,1973$.

[22] G.N. Lance and W.T. Williams. A general theory of classificatory sorting strategies. Computer Journal, 9:373-380, 1967.

[23] M. Luhn. The automatic creation of literature abstracts. IBM Inurnal nf Research and Denelopment. 2(2):159-165, April 1958.

[21] Y.S. Maarek. Using Structural Information for Managing lery Large Snfturare Sylstems. PhD thesis, Technion. Israel Institute of Technology, Haifa, Israel. January 1989.

[25] Y.S. Maarek. An incremental conceptnal clustering algorithm with input-ordering bias correction. In M.C. Golumbic, editor, Aduances in Artificial Intelligener. Natural Language and Knouledge Base. Systems. Springer Verlag, 1990.

[26] Y.S. Maarek and G.F. Kaiser. On the use of conceptual clustering for classifying reusable ada code. In Ada Leflers, Using Ada: ACM SIGAda International Conferener, pages 208-215, Boston. MA, December 1987. AC:N Press.

[27] Y.S. Maarek and F.A. Smadja. Full text indexing based on lexical relations. an application: Software libraries. In N.J. Belkin and C..J. van Rijsbergen, editors, Prnceedings nf SIGIR'89, pages 198-206, Cambridge. MA, June 1989. ACM Press. 
[28] W.J.R Martin. B.P.F. Al, and P.J.G. van Sterkenburg. On the prncessing of a text corpus: From textnal data to lexicographic information. In R.R.K. Hartmann, editor. Lexicngraphiy: Principles and Practice, London, 1983. Applied Language Studies Series, Academic Press.

[29] R. Michalski and R. Stepp. Antomated constructions of classifications: Conceptual clustering versus numerical taxonomy. IEEE Transactions on Pattern Analysis and Machine Intelligence, 5(4):396-409, July 1983.

[30] R. Prieto Diaz and P. Freeman. Classifying software for reusability. IEEE Softurare, 4(1):6-16, Jannary 1987.

[31] G. Salton. Antomatic text processing. the transformation, analysis and retrienal of information by computer. Addison-Wesley. Reading, MA, 1989.

[32] G. Salton and M.J. McGill. Introduction to Modern Information Retrienal. Computer Series. McGrawHill, New York. 1983.

[33] G. Salton and M. Smith. On the application of syntactic methodologies in antomatic text analysis. In Proceedings of SIGIR 89. pages 137-150. Cambridge. MA, June 1989. ACM Press.

[34] R.W. Schwanke. R.Z. Altncher, and M.A. Platoff. Discovering, visualizing and controlling software structure. In Proceedings of the Fifth International Workshop on Snfturar Srecifications and Design, pages 14i-150, Pittsburgh, PA, May 1989.

[35] F.A. Smadja. Lexical co-occurrence: The missing link. Journal of the Associntion for Literary and Linguistic Computing. Oxford University Press, 4(3), 1989.

[36] K. Sparck. Jones and J.I. Tait. Autornatic search variant generation. Journal of Documentation, 40(1):50-66. March 1984.

[37] W.F. Tichy. R.L. Adams, and L. Holter. NLH/E: A natural-language help system. In Proceedings of the $11^{\text {th }}$ ICSE. pages 364-374, Pittsburgh, PA, May 1989.

[38] C.J. van Rijsbergen. Information Retrienal. Butterworths, second erlition. 1979.

[39] M. Wood and I. Sommerville. An information retrieval system for software components. SIGIR Forum, 22(3,4):11-25, Spring/Summer 1988. 


\title{
An Information Retrieval Approach for Automatically Constructing Software Libraries
}

\author{
Yoëlle S. Maarek \\ IBM Thomas J. Watson Research Center \\ P.O. Box 704 \\ Yorktown Heights, NY 10598 \\ yoelle@ibm.com \\ Daniel M. Berry' \\ Technion, Israel Institute of Technology \\ Computer Science Department \\ Haifa, 32000, Israel \\ dberry@techsel.bitnet \\ Gail E. Kaiser \\ Columbia University \\ Department of Computer Science \\ New York, NY 10027 \\ kaiser@cs.columbia.edu
}

September 1990

CUCS-049-90

(C)1990 Yoëlle S. Maarek, Daniel M. Berry and Gail E. Kaiser. 


\title{
An Information Retrieval Approach for Automatically Constructing Software Libraries
}

\author{
Yoëlle S. Maarek \\ IBM Thomas J. Watson Research Center \\ P.O. Box 704 \\ Yorktown Heights, NY 10598 \\ yoelle@ibm.com
}

\author{
Daniel M. Berry \\ Technion, Israel Institute of Technc \\ Computer Science Department \\ Haifa, 32000, Israel \\ dberry@techsel.bitnet
}

\author{
Gail E. Kaiser \\ Columbia University \\ Department of Computer Science \\ New York, NY 10027 \\ kaiser@cs.columbia.edu
}

\begin{abstract}
Although software reuse presents clear advantages for programmer productivity and code reliabilit: it is not practiced enough. One of the reasons for the only moderate success of reuse is the lac of software libraries that facilitate the actual locating and understanding of reusable components This paper describes a technology for automatically assembling large snftware libraries that promot soltware reuse by helping the user locate the components closest to her/his needs.

Software libraries are automatically assembled from a set of unorganized components by usin infermation retrieval techniques. The construction of the library is done in iwo steps. First, attribute are automatically extracted from natural language documentation by using a new indexing scherr. hased on the notions of lexical affinities and quantity of information. Then, a hierarchy for browsin is alltomatically generated using s clustering technique that draws only nn the information provide by the attributes. Thanks to the free-text indexing scheme, tonls following this apprnach can accef free-style natural language queries.

This terhnology has been implemented in the Gunu system, which has been applied to construs an organized library of AIX utilities. An experiment was condurted in nreter in evaluate the retriev: effectiveness of GuRu as compared to INFOEXPLORER a hypertext library system fnr $\Lambda$ IX 3 nn the IBA RISC System $/ 6000$ series. We followed the ugual evaluation prorrlure usprl in information rntrieva based upon recall snd precision measures, and deternined that. our systrm performs $15 \%$ hetter on random test set, while being much less expensive to build than INFOF, xptorf.R.
\end{abstract}

Index Terms: automatic indexing, clustering, information retrieval, lexiral affinities, software 1 braries, soitware reuse. 


\section{Introduction}

Software reuse is widely believed to be a promising means for improving software productivity and reliability [14], and therefore is an issue of growing interest in software engineering. Unfortunately, not enough adequate libraries of reusable software components are available. By adequate, we mean that the library:

- provides a sufficient number of components, over a spectrum of domains, that can be reused as is (black-box rense) or easily adapted (white-box reuse), and

- is organized such that existing code closest to the users' needs is easy to locate. In particular, the library should provide mechanisms to help the reuser look for "finctionally close" components that meet some given requirements.

This paper is concerned with the second adequacy issue, and more generally with library systems that provide means for representing, storing and retrieving reusable components.

The first stage in building a library consists of indexing the objects to be stored in it, that is, producing a set of characterizing attributes, or signature, for each of these objects. The signature for each object represents the rensable object. Therefore, the quality of indexing is crucial to the quality of the library. Functionality is an important aspect of software components. Thus, it is necessary to include conceptual information about functionality in the indices. Unfortunately, conceptual information is difficnlt to obtain. Few programmers provide conceptual indices for their code. Moreover, even if provided, they can hardly be expressed nnder a common formalism since pieces of code typically originate from multiple sonrces. One solution is to manually index software components a posteriori according to a given classifying scheme, but this task is both arbitrary and tedious.

As an alternative, we propose to automatically identify indices by analyzing the natural-language documentation. in the form of manual pages or comments, usually associated with the code. Naturallanguage documentation is clearly a rich sonrce of conceptual information. However, this information is contained only implicitly, in an unstructured way, and is not usable as such. In order to extract usable information from free-style documentation, we propose to use information retrieval techniques. Once the indices have been produced, components can be automatically classified, stored and retrieved according to their signatures.

The classifying stage in the construction of a library consists of gathering objects into classes such that the members of the same class share some set of properties. The basic motivation for classifying is to facilitate browsing among similar components in order to identify the best candidates for rense. So that, during retrieval, a set of potentially adaptable components can be easily located. Browsing is more important for software libraries than for other kind of libraries, since there rarely exists a component perfectly matching a user's query. Moreover, local browsing allows the nser to discover unanticipated opportunities for reuse.

We have designed and implemented a tool, GurU, that embodies the above approach. GuRU automatically assembles conceptually structured software libraries from a set of unindexed and unorganized software components. In the first stage, GuRU extracts the indices from the natural language documentation associated with the software components to be stored, by using a new indexing scheme. This indexing scheme is based on lexical affinities and on their statistical distribution. It identifies a set of attributes for each document to represent a functional description of the associated soltware unit. In the second stage, GuRU assembles the indexed objects into a browsing hierarchy by using a hierarchical clustering technique that draws information exclusively from the indices identified in the previous stage. Thus, GuRU 
supports both classical linear retrieval. in which candidates are ranked according to a numerical measure that evaluates how weil they answer the query, and cluster-based retrieval in which the browse hierarchy directs the search for the best candidate.

Section 2 briefly compares the artificial intelligence and information retrieval approaches to constriction of software libraries and explains why we follow an IR approach. Section 3 describes the indexing method. Section 4 presents the classification approach and the clustering technique used for assembling the library. Section 5 deals with the retrieval stage. Section 6 gives results using onr GuRu implementation and a formal evalnation based on nsual methodology for evaluating information retrieval systems. Finally, Section 7 summarizes the main contributions of this work. Related work is discnssed as relevant throughout the paper.

\section{AI vs IR approach}

Previous efforts for building reuse systems can be roughly classified into two groups according to the approach adopted. the information retrieval (IR) approach or the artificial intelligence (AI) approach.

The IR approach consists of drawing information only from the structure of some documents that provide information on the software components. No semantic knowledge is used and no interpretation of the document is given: the reuse tool attempts to characterize the document rather than understand it. There are currently very few software library systems that follow an IR approach, or use existing IR techniques. Among them, the RSL system. [6] for instance, automatically scans source code files and extracts comments explicitly labeled for reuse with attributes such as keyword, anthor, date created, etc. The keyword attribute provides a list of free-text single-term indices very much like those used in IR tools. The Reuse system [3] provides a menu-driven front end to an information retrieval system, this all kind of soltware objects (inclinding user menus and system thesauri) are stored as textual docnments. This, the two previous systems use some kind of IR related technique, however the only system, to our knowledge, that applies a pnre IR approach is the system proposed by Frakes and Nejmeh [15]. They nse the CATALOG information retrieval system for storing and retrieving $C$ software components. Each component is characterized by a set of single-term indices that are antomatically extracted from the natural-language headers of $\mathrm{C}$ programs. Therefore, the construction of the $\mathrm{C}$ components repository is done antomatically, and does not require any pre-encoded knowledge as in Rst, for instance.

In contrast, in the $A$ I approach, the rense tool aims at understanding the queries and the functionality of components before providing an answer. Al-based systems are often smarter than IR systems. Some of them are context sensitive and can generale answers adapted to the user's expertise. As a tradeoff, they require some domain analysis and a great deal of pre-encorled semantic information, which is usually provided manually. They are based upon a knowledge base that stores semantic information about the domain and abont the language itself in case of a natural-language interfare. The main problem of applying this approach in the context of soltware libraries is that many domains cannot be easily circumscribed and the domain analysis is very difficult [10]. This makes the construction of such systems very tedious and expensive. Examples of AI or knowledge-based reuse tools are numerous, e.g. [30], [39], [2], [11], [37]. of

The AI approach can be iseful in some applications. However, we prefer the IR approach for reasons

- cost: the library system is built entirely automatically,

- transportability: the library system can be rebuilt for any domain since it does require manually 
provided domain knowledge,

- scalability: the repository can be easily updated when new components are inserted, either by recompiling the indices or by applying incremental techniques, the indexing task is entirely mechanical.

We therefore propose to apply a pure IR approach, in the same direction of research as Frakes and Nejmeh, by automatically building free-text indices that characterize soitware components. We also propose to use an indexing scheme richer than the single-term indexing used in the IR-based tools described in this section so as to achieve a better retrieval effectiveness. The following section explains our source of information and how the indexing is performed.

\section{The Indexing Stage}

The major advantage of automatic indexing over manual indexing, besides the obvious cost considerations, is that it allows a unified scheme, insuring that indices will be compatible with each other. The idea is to extract attributes from an existing source of information, i.e., the corle and the natural-language documentation. Some work has been done towards extraction of primitive functional information from the code [26]. [34], however, the richer source of functional information is the natural-language documentation, assuming any is available.

An examination of numerous samples of code allowed us to reach the conclusion that some nseful information can be extracted from programs written in a high-level language using good programming style. whereas little conceptual information can be found in typical real-world code chosen at random [24]. Unfortunately, even when dealing with well-written code, there is a very low probability that the programming styles of the various pieces of code will be consistent. Even a.single programmer may use totally different identifiers for expressing the same concept from one day to another. Since software components come from multiple sources in the context of large software libraries, extracting autributes from code would necessitate as many indexing schemes as there are code sonrces. Another limitation comes from the fact that there are many more possibilities for identifiers than for natural-language words since they do not follow any morphological or syntactic rules.

In other words, when there is no way to guarantee good, and let alone consistent and compatible, programming styles, extracting attributes from raw code does not give significant results. Therefore, we prefer concentrating on the other possible sonrce of information, i.e., the natnral-language documentation either inserted into the code, i.e., the comments, or associated with the code, e.g., manual pages.

Comments are intended to help programmers understand the cole and thus may provide functional information. They deal with specific parts of the code into which they are inserted, and they may give information on various parts at various levels of abstraction. Fxtracting functional information from comments entails two activities,

- defining an indexing scheme that allows extracting attributes from natural language phrases or sentences, and

- relating comments to the portion of code they concern.

The second activity is very complex in free-style code. Indeed, in free-style programming, programmers can insert comments wherever, and in any format and any length, they wish. Although comments usnally describe the containing routine or the one just below, in general it is impossible to antomatically determine 
what part of the code is covered. A solution would be to consider that all the comments inserted in a specific piece oi code constitnte a global natural-language description of the considered code. Unfortunately, this is not the case. Comments rank from low-level implementation details to high-level description. For instance, in the rm. $c$ source file in Berkeley UNIX, one can find comments as varions as:

1. current pointer to end of path $*$, or

1. rm - for ReMoving files, dizectories trees. *I

The first conveys no useful functional information while the second hits the mark exactly. In general, there are many more low level, and useless for our purpose, comments than high level ones. and there is no way to automatically distinguish between them. Therefore, so long as no style is enforced, it is very difficult to extract useful information from comments.

Let ns note, however, that any piece of natural language, from comments inserted in the code to design specifications, which is specifically related to software code and whose level of abstraction is known can bring nseful information. Thus, we are currently working on extracting functional information from comments in the framework of RPDE [1T], a structured software development environment. in which comments are linked to the portion of code they describe. In the following, though, we try to remain as general as possible, and we do not assume that any commenting style is enforced. Therefore, although our indexing scheme is applicable to any piece of natural-language that brings some functional information, we will exemplify it through the analysis of manual pages clearly related to reusable components, such as UNIX-like manual pages.

In the rest of this paper, the $A \mid x$ documentation is taken as our corpus since it fulfills the requirement of being structured into manual pages. Moreover the AIX docnmentation can be seen as a regular realworld documentation database since it is of average quality as far as commenting style is concerned. Many even consider the AIX documentation of poor quality when compared to Berkeley UNIX documentation due to typos, inconsistent style, poor vocabulary, etc.

\subsection{A Richer Indexing Unit: the Lexical Affinity}

There has been much work in IR dealing with natural-language text, a large variety of techniques have been devised for indexing, classifying and retrieving documents [31]. One of the main concerns in IR is the aut tomatic indexing of documents, which consists of producing lor each document a set of indices that form a signature of the document. A signature is a short-form description of a tocument, easier to manipulate than the entire document, which plays the role of a surrogate at the retrieval stage.

Several issues need to be addressed when indexing a document with respect to the nature and the form of the produced indices. More precisely, the indexing vocabulary can be either controlled or uncontrolled. In the controlled vocabulary approach only a restricted set of indices are all thorized (e.g.. in MEDLARS [32]), whereas in the uncontrolled vocabulary, or free text, approach, there is no constraint on the nature of the indices. It has been shown that both approaches are comparable in terms of performance, [14], [32], however we prefer the uncontrolled vocabulary approach in the context of software reuse, for the same reasons of cost, portability and scalability. Indeed, defining an adequate controlled vocabnlary is a mannal, domain-dependent task and, therefore, suffers from the same drawbacks as the encoding of a knowledge-base.

Another important issue in automatic indexing deals with the natnre of the indices. The most usual form is single-term index, in which single words without contextual information are selected as indices. Unfortunately, single term indices are often too specific or too broad and can induce ambiguities. Therefore, 
it has been proposed to take term phrases as indexing units rather than single terms so as to refine the meaning of constituent words. However, the use of word co-occurrences has not brought good results.as expressed by Salton [31] (p 296):

"... a phrase-formation process controlled only by word co-occurrences and the document frequencies of certain words is not likely to generate a large number of high-qquality phrases."

As an answer to this problem, a possible solution has been to add syntactic criteria in order to provide further control in phrase formation, such as part-of-speech using specially formatted dictionaries [21], or more refined analysis inchiding semantics [36]. But,

"The available options in phrase generation appear limited, and the introduction of costly and refined methodologies may bring only marginal improvements." [31] (p 298)

We are more optimistic, and believe that indexing units richer than single terms can be 1 sed and bring significant improvement at low cost. The atomic unit we propose to use in order to demongtrate this is derived from the notion of lexical affinity. In linguistics, a syntagmatic lexical affinity (LA), also termed lesical relation. between two units of language stands for a correlation of their common appearance in the utterances of the language [8]. The observation of LAs in large textual corpora has been shown to convey information on both syntactic and semantic levels, and provides us with a powerfnl way of taking context into acconnt [35].

We propose to use the notion of LA for indexing purposes, and restrict the above definition by observing LAs within a finite document rather than within the whole language so as to retrieve conceptual affinities that characterize the document', rather than purely lexical ones. Moreover, we only consider LAs involving open-class words as meaning-bearing, whereas LAs involving closed-class -words ${ }^{2}$ are not.

Ideally, LAs are extracted from a text by parsing it since two words share a lexical affinity if they are involved in a modifier-modified relation. Unfortunately, antomatic syntactir parsing of free-style text is still not very efficient [33]. Instead, we make use of simple co-occurrence. It has been shown by Martin et al. that $98 \%$ of lexical relations relate words that are separated by at most five words within a single sentence [28]. Therefore, most of the LAs involving a word $w$ can be extracted by examining the neighborhood of each orcurrence of $w$ within a span of five words $(-5$ words and +5 ivords around $w)$.

The extraction technique consists of sliding a window over the text and storing pairs of words involving the head of the window (if it is an open-class word) and any of the nther open-rlass elements of the window. The window is slid word by word from the first word of the sentence to the last. the size of the window decreasing at the end of the sentence so as not to cross sentence boundarins'. siner lexical affinities cannot relate words belonging to different sentences. The window size being smaller than a constant, the extraction of $\mathrm{LAs}$ is linear in the number of words in the document. An algorithm for the sliding window technique is presented in Figure 1. Maarek and Smadja have used a similar technique in [2i], which was also based on Martin's results [28], but more adapted to the analysis of large corpora.

In summary, the first stage in indexing a manual page consists of extracting all the potential LAs by using the sliding window lechnique, and storing them under their canonical form, in which each word is represented by its inflectional root (or lemma). An example of the potential LAs extracted from the

\footnotetext{
'rather than the whole language.

${ }^{2}$ In general, open-elass words include nouns, verbs, adjectives and adverbs, while closed-elass words are pronouns, prepositiong, conjunctions and interjections.

${ }^{3}$ The isolation of sentences is the only parsing performed.
} 


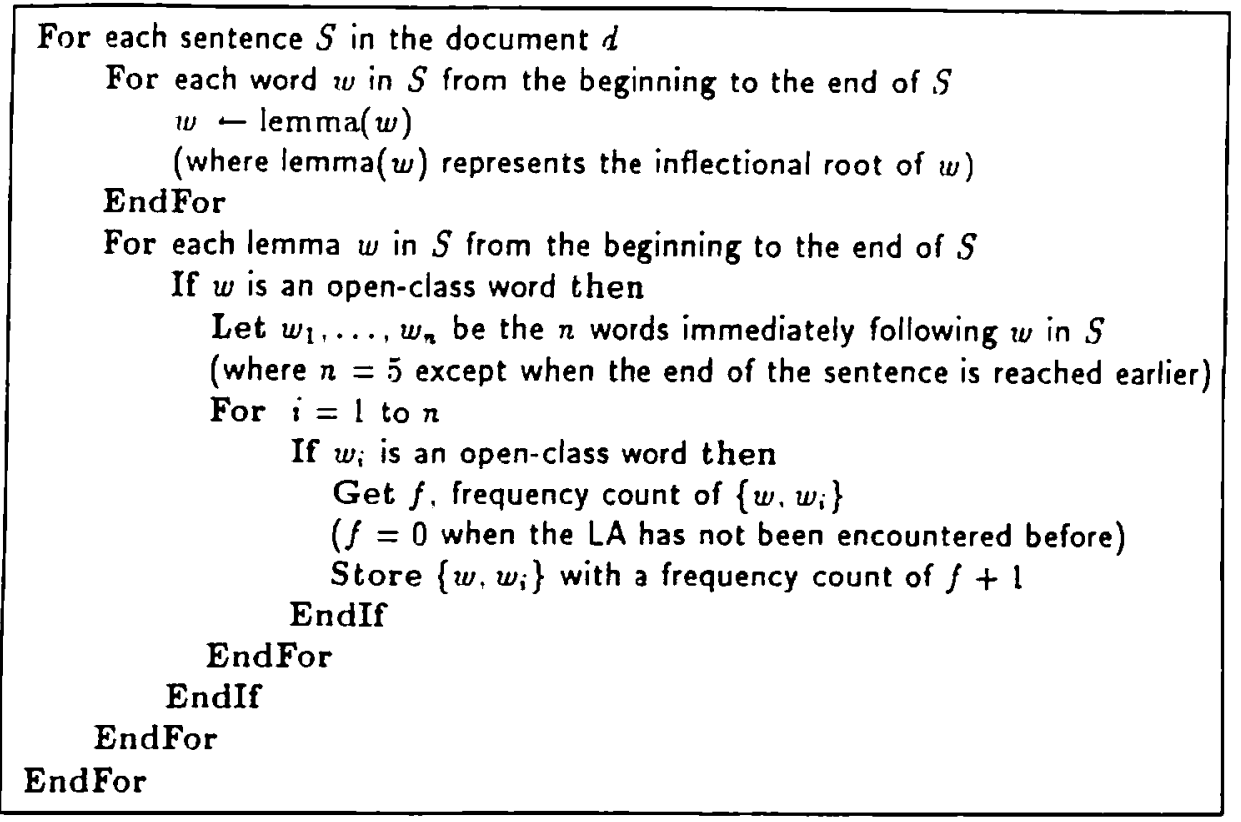

Figure 1: Sliding window technique

mannal page of $m v$ in $A I X$ and ranked by frequency of occurrence are presented in Table 1 . For the sake of the comparison, a list of the single words extracted from the same manual page is shown in the first column, also ranked by frequency of appearance.

$\Lambda$ mong the extracted lexical relations, some correspond to abstractions of the considered document. and some do not. Since we are interested in indexing textual documents, in the first stage, we isolate actual affinities by using frequency criteria. It has been demonstrated that the frequency of occurrence of a term within a document is related to the importance of the word in a text [23]. This is also true for the common appearance of pairs of words and even more for lexical affinities.

\subsection{From LAs to Indices}

When analyzing a document, many potential lexical affinities are thus identified. Some of these lexical affinities are conceptually important and some are not. As seen in Tahle 1. frequency of appearance is a good indicator of relevance. However, some noise exists, mainly dine tn wnrrls appearing ton often in a given context. In order to reduce the influence of such words, it is necessary in the second stage to select from among the lexical affinities identified only the most representative ones, i.e., those containing the most information.

We have defined a measure evaluating the resolving power of an LA. It is based upon the quantity of information of each of the words involved in the LA, as well as upon the frequency of appearance of this LA within the considered document. The quantity of information of a word within a corpus is defined as:

$$
\operatorname{INFO}(w)=-\log _{2}(P\{w\})
$$

where $P\{w\}$ is the observed probability of occurrence $w$ in the corpus [4], [32]. Therefore, the more 


\begin{tabular}{|l|l||l|l|}
\hline open-class words & freq & LAs & freq \\
\hline file & 30 & file move & 9 \\
directory & 14 & be file & 8 \\
mv & 11 & directory file & 7 \\
files & 8 & file system & 5 \\
new & 7 & file overwrite & 5 \\
name & 7 & file mv & 5 \\
move & 7 & file name & 4 \\
newname & 6 & name path & 3 \\
is & 6 & do file & 3 \\
system & 5 & directory move & 3 \\
one & 5 & different file & 3 \\
$\ldots$ & $\ldots$ & $\ldots$ & $\ldots$ \\
\hline
\end{tabular}

Table 1: Keywords and lexical affinities classified by frequency in the mv mannal page

frequent a word is in a domain, the less information it carries. From this definition, we infer the definition of the quantity of information of an LA $\left(w_{1}, w_{2}\right)$ as:

$$
\operatorname{INFO}\left(\left(w_{1}, w_{2}\right)\right)=-\log _{2}\left(P\left\{w_{i}, w_{2}\right\}\right)
$$

To simplify the computation of this factor, in the rest of this work, we consider words within the textual universe as independent variables". Thus, we use the following formula for computing the quantity of information of an LA.

$$
\operatorname{INFO}\left(\left(w_{1}, w_{2}\right)\right)=-\log _{2}\left(P\left\{w_{1}\right\} \times P\left\{w_{2}\right\}\right)
$$

Then, we define the resolving power of an LA in a given document as follows. Let $\left(w_{1}, w_{2}, f\right)$ be a tuple retrieved while analyzing a document $d$, where $\left(w_{1}, w_{2}\right)$ is an $\mathrm{L} A$ appearing $f$ times in $d$. The resoluing power of this LA in $d$ is defined as:

$$
\rho\left(\left(w_{1}, w_{2}, f\right)\right)=f \times \operatorname{INFO}\left(\left(w_{1}, w_{2}\right)\right)
$$

The higher the resolving power of a lexical affinity is, the more characteristic of the document it is. The resolving power allows us to evaluate the importance of a lexical affinity within a text by taking into account both its frequency of appearance in the text and the quantity of in formation of the words involved. Thus, even though the lexical affinity (be file) appears very often in an AIX manual page, it has only a small resolving power, simply because the quantity of information of hoth thr words "file" and "be" in the $\Lambda$ IX documentation is low.

In order to be able to compare the relative performances, in terms of resolving power, of different documents, we transform the raw $\rho$ score into a standardized score. The standardized score, or z-score, is defined as $\rho:=(\rho-\bar{\rho}) / \sigma$ where $\bar{\rho}$ and $\sigma$ are the average and standard deviation of the $\rho$-values. This transformation does not alter the distribution and allows us to evaluate the relative status of the score in the $\rho$ distribution. In the rest of this paper, the $\rho$-values we give as examples will therefore represent the z-score rather than the raw srore.

\footnotetext{
'This assumption represents only an approximation since words in English are definitely not independent, but are distributed according to the rules of the language.

${ }^{5}$ This notion is related to that of mutisal information [4].
} 
Table 2 compares the list of LAs for the mv manual page ranked by frequency and by resolving power. In it, the LA (file move) has a greater.resolving power than any of the following LAs. Moreover, some noisy LAs such as (do file) or (be file) (in italic fonts in the table) have disappeared because both words involved in the LAs are highly frequent in the corpns and thns have a low quantity of information.

\begin{tabular}{|l|l|}
\hline LAs & freq \\
\hline file move & 9 \\
be file & 8 \\
directory file & 7 \\
file system & 5 \\
file overwrite & 5 \\
file mv & 5 \\
fle name & 4 \\
name path & 3 \\
do file & 3 \\
directory move & 3 \\
\hline
\end{tabular}

\begin{tabular}{|l|l|}
\hline LAs & $\rho$ \\
\hline file move & 8.38 \\
file mv & 4.36 \\
directory file & 4.03 \\
file overwrite & 3.87 \\
directory move & 1.98 \\
file system & 1.95 \\
mv rename & 1.71 \\
move mv & 1.58 \\
different file & 1.40 \\
name path & 1.33 \\
\hline
\end{tabular}

Table 2: Comparison of frequency and $p$-value for the LAs in mv

For each document, we select as indices those LAs with the highest resolving power. More precisely, we are interested in the LAs that represent peaks in the distribution of $\rho$-valıes. Therefore, we keep as indices only the LAs whose $\rho$ value is one standard deviation above the mean, i.e., such that $\rho \geq \bar{\rho}+\sigma$, where $\bar{\rho}$ represents the mean and $\sigma$ the standard deviation of the distribution of $\rho$ values within one document. The choice of such a threshold ${ }^{6}$ is reflected in Tables 2, 3 and 4 , where only LAs with a z-score greater than 1 are presented.

The set of LAs of a document selected by ranking $\rho$-values and taking those one standard deviation above the mean forms the signatnre of the docnment. The major contribution of this technique consisted in adapting the notion of lexical affinity for indexing purposes. We gave some intuitive indications on how an $L A$-based indexing scheme is richer than a single-word scheme. We will demonstrate later that it ensures a better retrieval effectiveness.

The next section explains how software components can be stored and classified using the signatures produced at the indexing stage.

\section{The Classifying Stage}

Normally, when a user wants to use a software library, s/he first has to arress a library that might contain the desired component, then has to provide a formal description of the researched component according to the vocabulary understood by the library system. Unfortunately, in most cases, this ideal scenario does not work out. The majn reason is that in real life applications, the component perfectly matching the nser's requirements does not exist in the library, or it is not indexed as the nser had guessed it would be.

In such cases, a traditional database management system fails to help the user. Indeed, to be retrieved from the database, a component must exactly match the query ${ }^{7}$. Such strict matching is inappropriate

\footnotetext{
"This classical threshold guarantees to keep only a small percentage of the sample eiements in most digtributions.

'A notable exception is ARES [18], a relational database that allows flexible interpretation of queries. In ARES the
} 


\begin{tabular}{|l|l|}
\hline LAs & $\rho_{z}$ \\
\hline copy file & 6.49 \\
directory file & 2.47 \\
file source & 2.15 \\
infile subdirectory & 1.98 \\
contain subdirectory & 1.30 \\
copy cp & 1.30 \\
copy regular & 1.02 \\
\hline
\end{tabular}

Table 3: LAs ranked by p-values for cp

\begin{tabular}{|l|l|}
\hline LAs & $\rho$ \\
\hline directory make & 5.08 \\
create mkdir & 2.74 \\
directory mkdir & 2.55 \\
directory permission & 1.48 \\
directory write & 1.03 \\
\hline
\end{tabular}

Table 4: LAs ranked by $p$-valnes for mkdir

in a software library system since the user often cannot know the exact characteristics of the desirable component and, even when $\mathrm{s} / \mathrm{he}$ does, there is rarely a perfect match.

Software libraries should not only permit retrieving candidate components that perfectly or partially match the query, but also permit browsing among components that share some functionality. It is therefore desirable to structure the library for making the search, retrieval and browsing mechanisms as fast and convenient as possible. in order to make the access to the library attractive.

We propose here to perform the search and retrieval operations using a conventional inverted index file structure, and to cluster the library in order to facilitate the browsing operation. Section 4.1 explains how the infex repository is brilt using an inverted file structure, and Section 4.2 presents the clustering technique used to build the browse hierarchy. Section 5 explains how they are used to perform the search and browsing operations.

\subsection{Building the index repository}

The goal is to allow a fast and easy identification of candidate components at the retrieval stage. This, we derive from the signature repository built at the indexing stage another repository for storing, for each word, the LAs involving that word, and pointers to the docnments in which it appears. Let us denote:

- $W$ the universe of words

- $\mathcal{D}$ the universe of documents.

Index LAs are defined as tuples $\left(w, w^{\prime}, \rho\right)$ where $w$ is smaller than $w^{\prime}$ in the lexicographic order and $\rho$ is the resolving power of this LA in a considered document. The reason for ordering $w$ and $w^{\prime}$ is to avoid

similarity between elements can be evaluated via a lookup in a table that has to be provided beforehand. ARES is not discussed here since its purpose is not to elassily software. Further, it has the drawback of requiring a great deal of preencoded knowledge. 
duplicate LAs by forcing every LA into a canonical form.

The index stored in the repository is represented as a mapping defined as follows:

$$
w \in \mathcal{W} \rightarrow \lambda(w)=\{(x, \rho, d) \in \mathcal{W} \times[1, \infty[\times \mathcal{D} \mid \text { either }(w, x, \rho) \text { or }(x, w, \rho) \text { is an } \operatorname{LA} \text { of } d\}
$$

The mapping $\lambda$ is stored as a trie data structure. The mapping $\sigma$ between documents to their signatures is also stored using a trie data structure:

$$
d \in \mathcal{D} \rightarrow \sigma(d)=\left\{\left(w, w^{\prime}, \rho\right) \in \mathcal{W}^{2} \times\left\{1, \infty \|\left(w, w^{\prime}, \rho\right)\right\} \text { is an LA of } d\right\}
$$

In implementing these mappings, tries are usually faster than hashing schemes, although they consume more memory. In this case, fast access is a basic requirement for making the retrieval stage attractive. These two mappings are the basic operations we use to retrieve and rank candidates as explained in Section 5 .

\subsection{Building the browse hierarchy}

As explained previously, browsing is crucial in software library systems. The most common way to make browsing operations possible is to group items judged to be similar by using clustering operations [31]. Jardine and van Rijsbergen [19] pointed out that "associations between documents convey information about the relevance of docllments to requests". They demonstrated that cluster-based retrieval strategies are as effective as linear strategies and much more efficient. Thus, many clustering methods have been used for information retrieval [19], [7], [16]. The most popular clustering methods are the hierarchical ag. glomerative clustering (HAC) methods because their search and construction techniques are more efficient than for most non-hierarchical methods [19].

The following sections define some terminology in cluster analysis, describe the algorithms we used to build the browse hierarchy, and present some samples of the browsing hierarchy obtained for the AIX library.

\subsubsection{Some terminology in cluster analysis}

Classification by cluster analysis has been of long-standing interest in statistics as well as various other fields. It can be traced back to the work of Adanson in $1757[1]$, who used numerical clustering for classifying botanic species. Statisticians and taxonomists have widely developed the field since then. Clister analysis now offers a wide range of techniques for identifying nnderlying strnctures in large sets of objects and revealing links between objects or classes of objects. One particular appliration of classification is the building of libraries.

There is no strict definition of cluster, but it is generally agreed that $n$ cluster is a group of objects whose members are more similar to each other than to the members of any other group. Typically, the goal of cluster analysis is to determine a set of clusters, or a clustering, such that inter-cluster similarity is low and intra-cluster similarity is high. The similarity between objects is evaluated via a numerical measure called a dissimilarity index defined as follows.

Definition 1 Let $\Omega$ be a set of objects. A dissimilarity index $\delta$ over $\Omega^{2}$ is a function from $\Omega \times \Omega$ to $R_{+}$ that satisfies the following properties,

$$
\text { (i) } \forall 0 \in \Omega, \delta(0,0)=0 \text {, }
$$


Note that a distance is a dissimilarity inder hut that a dissimilarily index does not necessarily satisfy the triangle inequality and therefore is not a distance.

The dissimilarity index between objects is used as the basic criterion to determine clusters. Clustering techniques allow identifying not only clusters but also relationships among them. The structure of the set of clusters as well as their internal structure vary with the clustering technique. Clustering methods are usnally classified ${ }^{8}$ according to the structure of the set of clnsters produced, e.g, hierarchical. flat, overlapping, etc., as well as the technique used, e.g.. divisive, agglomerative, incremental, etc. As explained previonsly, hierarchical agglomerative techniques are very convenient for building browse hierarchies. The basic principle that these techniqnes follow is presented below.

Hierarchical numerical clustering aims at building hierarchies, over a set of objects, in which each internal node corresponds to a clister of objects and each leaf represents an individual object, or more precisely a singleton cluster. Most hierarchical clustering methods are based npon the same general method, called the Hierarchical Agglomerative Clustering (HAC) method [12], which consists of iteratively gathering objects into clusters, until only one cluster remains.

The HAC general method iteratively bnilds a sequence of partitions or level clusterings of $\Omega$, that is. a sequence of disjoint clusters covering the original set of objects, $\Omega$. The level clusterings form coarser and coarser partitions by an iterative process, beginning with the level clnstering formed by the set of singletons in the power set $\rho(\Omega)$, i.e., $\left\{\left\{o_{1}\right\},\left\{o_{2}\right\}, \ldots,\left\{o_{n}\right\}\right\}$, and ending up with the coarsest partition of $\Omega$, i.e., $\{\Omega\}$. The final output of this clustering process is a particular form of hierarchy called a derdogram. The HAC general method can be expressed as follows:

- Start with the subset of $p(\Omega)$ formed by singleton elements.

- Repeat the following steps iteratively until there is only one cluster.

- Identify the two clusters that are the most similar.

- Merge them together into a single cluster.

The HAC method requires a measure of similarity not only over the set of objects, but also over the set of clusters. The dissimilarity index between clusters is usually derived from a user-given dissimilarity index, $\delta$, between objects. The way of defining $\Delta$ has a direct influence on the final form of the hierarchy obtained. Once a dissimilarity index $\delta$ between objects is provided, HAC methnds differ only by the choice of this measure. The most commonly used HAC methods are the single link and complete link methods [22]. Many other methods such as the centroid method. Ward's method, etr... refine still other dissimilarity indices but most of them require the dissimilarity index over $\Omega$ to he a distance, that is, to satisly the triangle inequality. The reader should consult [13] [12] for an extensive survey of the HAC. methods. The time complexity of the HAC algorithm is at most $O\left(n^{2} \log n\right)$ where $n$ is the number of objects involved. For some particular definitions of $\Delta$, it can be reduced to $O\left(n^{2}\right)$.

\footnotetext{
With the recent introduction of conceptual clustering (29), another distinction has been introduced according to the definition of the elusters obtained, in extension (i.e., by enumeration of its members) for regular (or numerical) clustering and in intension (1.e., by membership sules) as well as in extension for conceptual clustering.
} 


\subsubsection{Adapting a clustering technique for building a browse hierarchy}

As explained above, we propose to use a $\mathrm{HAC}$ technique to generate a browse hierarchy. In this perspective, we (1) need to define a measure of similarity between the objects considered, e.g.. the documents, and (2) explain how to make a browse hierarchy ont of the dendogram generated by the HAC technique. Let is address these two points.

In information retrieval, numerous measures of similarity between documents, also termed measures of association or coefficients of association, have been defined. The simplest of all is defined as:

$$
|X \cap Y|
$$

where $X$ and $Y$ are the signatures of two documents. This measure represents the number of common index nnits. Varions other measures [38] have been defined such as:

$$
\begin{array}{ll}
\frac{2|X \cap Y|}{|X|+|Y|} & \text { Dice's coefficient } \\
\frac{|X \cap Y|}{|X \cup Y|} & \text { Jaccard 's coefficient } \\
\frac{|X \cap Y|}{|X| \times|Y|} & \text { Salton's Cosine coefficient }
\end{array}
$$

They can all be considered as normalized versions of (9) since they are functions of the cardinality of $X, Y, X \cap Y$, or $X \cup Y$.

In our context, we have more information than just the presence or absence of index units in the signature, and therefore we propose to take into account the $\rho$-values of LAs in the evaluation of the measure of association between documents. For any signature $X=\left\{\left(w, w^{\prime}, \rho\right)\right\}, p(X)$ is the projection set of $X$ over $W^{2}$. Then, the simplest measure is $|p(X) \cap p(Y)|$. In order to take into account the resolving power of LAs as well, we define our measure $\delta$ for two signatures $X$ and $Y$, such that $X \neq Y$, as

$$
\delta(X, Y)=\sum_{\left(w, w^{\prime}\right) \in \in_{P}(X) \cap_{p}(Y)}\left(\rho X\left(w, w^{\prime}\right)+\rho_{Y}\left(w, w^{\prime}\right)\right)
$$

where $\rho_{X}\left(w, w^{\prime}\right)$ is the $\rho$ value of the LA $\left(w, w^{\prime}\right)$ in the signature $X$, and similarly for $Y$. Note that $\delta$ is a measure of similarity rather than a measure of dissimilarity. Its inverse is a measure of dissimilarity as long as $\delta(X, X)$ is set to a sufficiently large arbitrary value so that its inverse can be considered essentially null.

Given such a measure of similarity between signatures, we define a measure of similarity between clnsters according to the single link or complete link techniqnes for instanre and then nse the hierarchical agglomerative clnstering algorithm in order to build a browse hierarchy of snftware components. Let us note that we also made some experiments in earlier versions of Gunu using an incremental conceptual clustering technique [25] for constructing the browse hierarchy. However. despite interesting results, the cost of building and maintaining the hierarchy was prohibitive (exponential time like for most conceptual clustering techniques) when compared to regular clustering techniques and did not appear to be better in terms of retrieval effectiveness.

All the HAC techniques build a binary hierarchy. Not all levels of the hicrarchy are equally significant: therefore. the "sual approach is to select manually the most significant level clusterings, this task being usually performed by a data analyst. The following proposes a method for antomatically identifying the most useful level clusterings, and thus producing a not-necessarily binary hierarchy.

This method of selection is based on the following principle. Each level clnstering in the dendogram corresponds to the merging of two clusters in the previous level clustering and therefore to a particular 


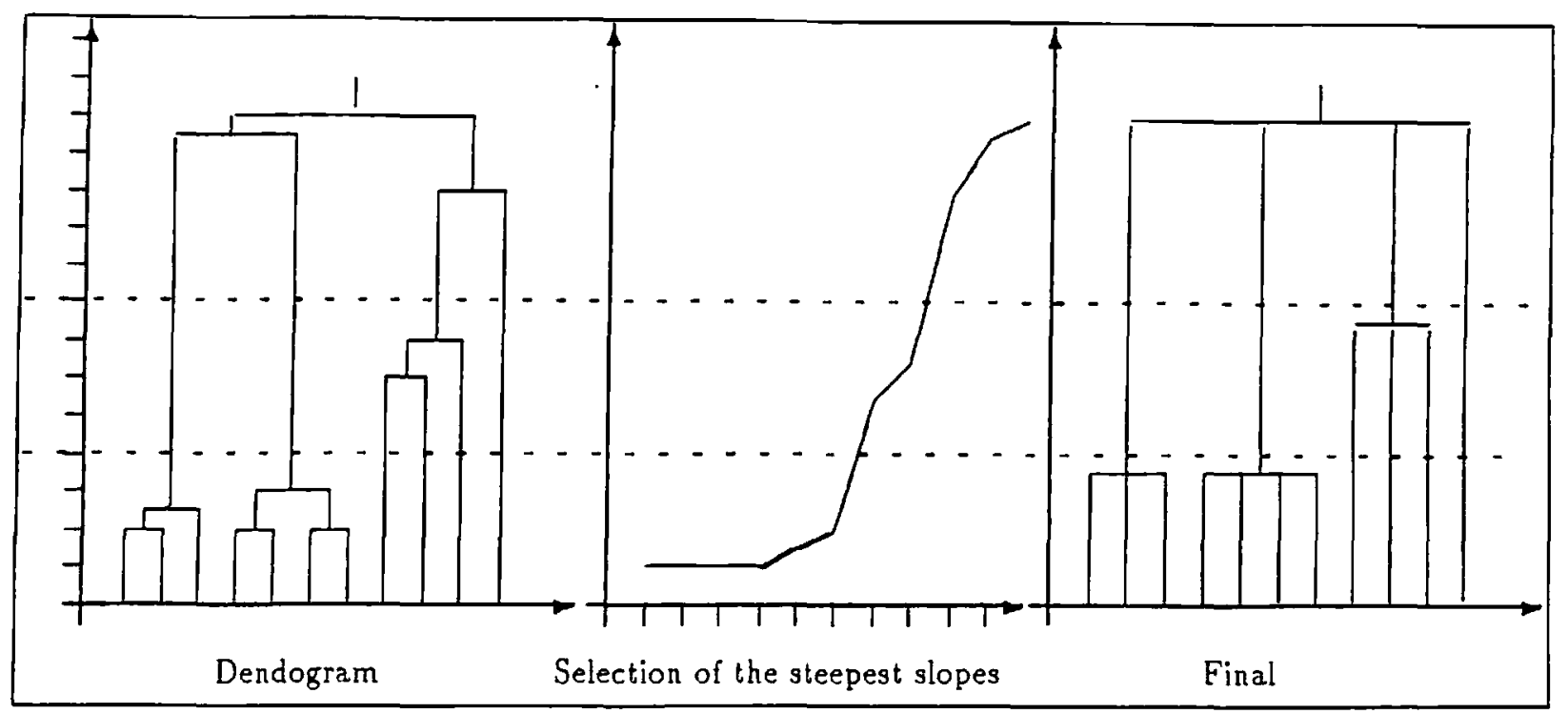

Figure 2: Principle of selection of level clusterings

value of the similarity measure. If we label the dendogram with these values $y_{n}, \ldots, y_{1}, n$ being the number of objects. from the bottom to the top of the hierarchy, it can easily be shown that the $y_{i}$ 's are (non-strictly) monotonic (increasing for dissimilarity measures and decreasing for similarity measures) for the single and complete link clnstering methods. We propose to select those levels that correspond to the gap in the distribution of $y_{i}$ 's by (1) plotting the segment connecting the pairs $y_{i+1}, y_{i}$ from $i=n-1$ to $i=1$, and (2) keeping the levels that correspond to the steepest slopes. This represents the intuitive method that a data analyst would apply. Figure 2 gives an intuitive presentation of the method via $a n$ example whereas Figure 3 gives the formal algorithm. The time complexity of the latter is linear in the number of objects.

\subsection{Some examples}

Portions of the browse hierarchy built from the AIX documentation are shown in Figures 4 and 5 . In Figure 4, some interesting clusters are isolated. Thns, in the figure we have a cluster gathering commands related to the manipulation of regular expressions, and a cluster gathering editors. These two clusters are also part of the same super-cluster, mainly because these editors permit to manipulate regilar expressions. Then, there are two outliers that could not be inclnded in a cluster: makekey and termdef. Then a small cluster groups ps and kill, which both are strongly related as they give information about processes or handle them. Finally, there are two big clnsters, one for yellow pages commands and another for SCCS routines. The clustering is not always of such good quality as can be seen in Figure 5, either becanse of the nature of the documentation or because of the principle of clustering itself. For instance, the commands $x c a l c$ and dc, which both are calculators, belong to a same cluster. but be has been forgotten in this clnster. This is due to the fact that the manual page of be does not refer to the concept of calculator at all. but defines $b c$ as an interpreter for an arithmetic language. The real problem with clnstering is illustrated with the third cluster in this fignre, which gathers batch, at. crontab, date and istat. This cluster has been formed because all these commands are related to the notion of date or time; unfortunately, this is not the main functionality of all of these commands and therefore this clister is somehow misleading. Let 


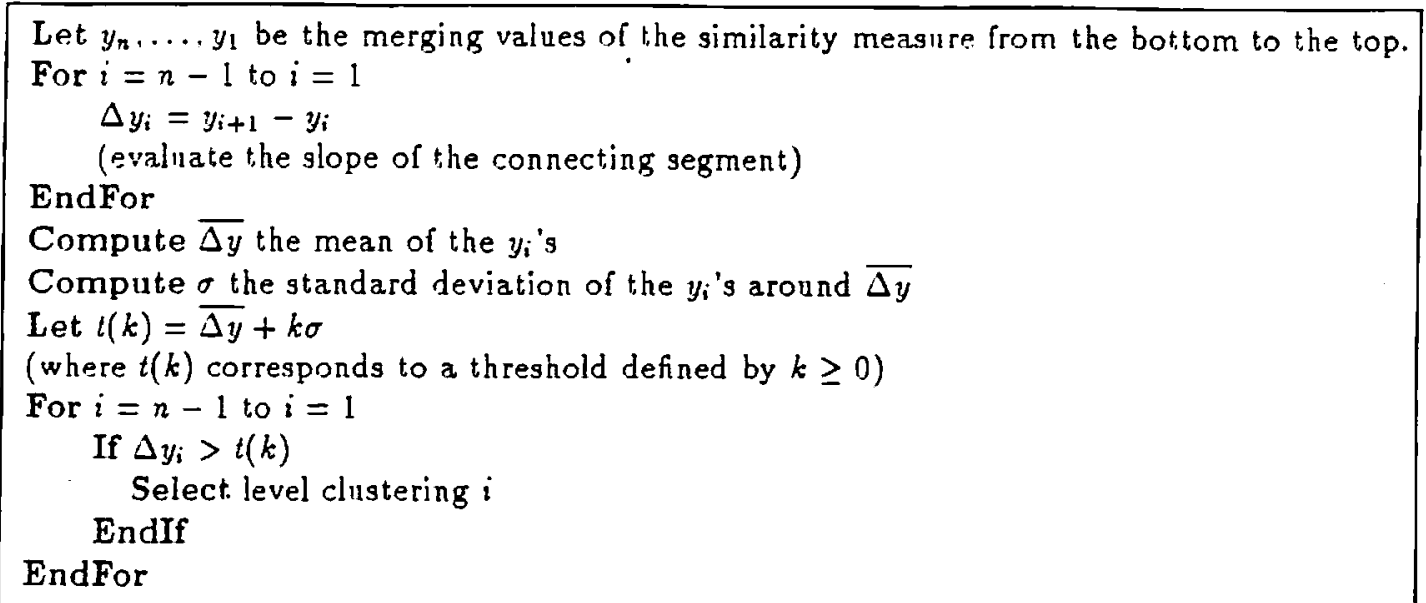

Figure 3: Selection of level clusterings

11.s note, however, that the lower level cluster including at and batch is a good one.

The hierarchy this generated is used as an aid to browse when nothing relevant has been retrieved via linear retrieval, or in order to increase recall since there is no way to be sure that all the relevant components have been retrieved at the linear retrieval stage. It can also be used as the basic repository to be searched during retrieval, but we prefer to use the traditional linear retrieval technique instead because it is clearly more trustable considering the problems described above.

By nature this indexing technique suffers from noise since it is based on only statistical observations. Noisy indices involve generally misspelled or un meaningful strings of characters that are mixed with natural langrage (for describing instructions lor instance), or "side-concepts" such as the time, day and month in the example rited above. This noise cannot be avoided when dealing with free-style text.

Fortunately, these noisy LAs do not cause real trouble at the linear retrieval stage since there is a very low probability that the user would use nnmeaningful character strings in her/his queries. So noisy LAs are part of the signatures of components but rarely lead to the selection of the considered component. On the other hand, noisy LAs might induce the formation of poor quality clusters, but generally only higher levels of the hierarchy are affected since "side concepts" are not given much weight when evaluating similarity. Section 5.3 explains how this browsing hierarchy is used at retrieval stage.

\section{The Retrieval Stage}

The previous sections explain how libraries of reusable components are assembled. We also need to be able to retrieve the components that match the requirements when at least one exists, or to assist in the selection of the closest components via a browsing facility.

The usual scenario when retrieving a component is the following:

- Query specification: The user expresses a query according to the anthorized vocabulary. 


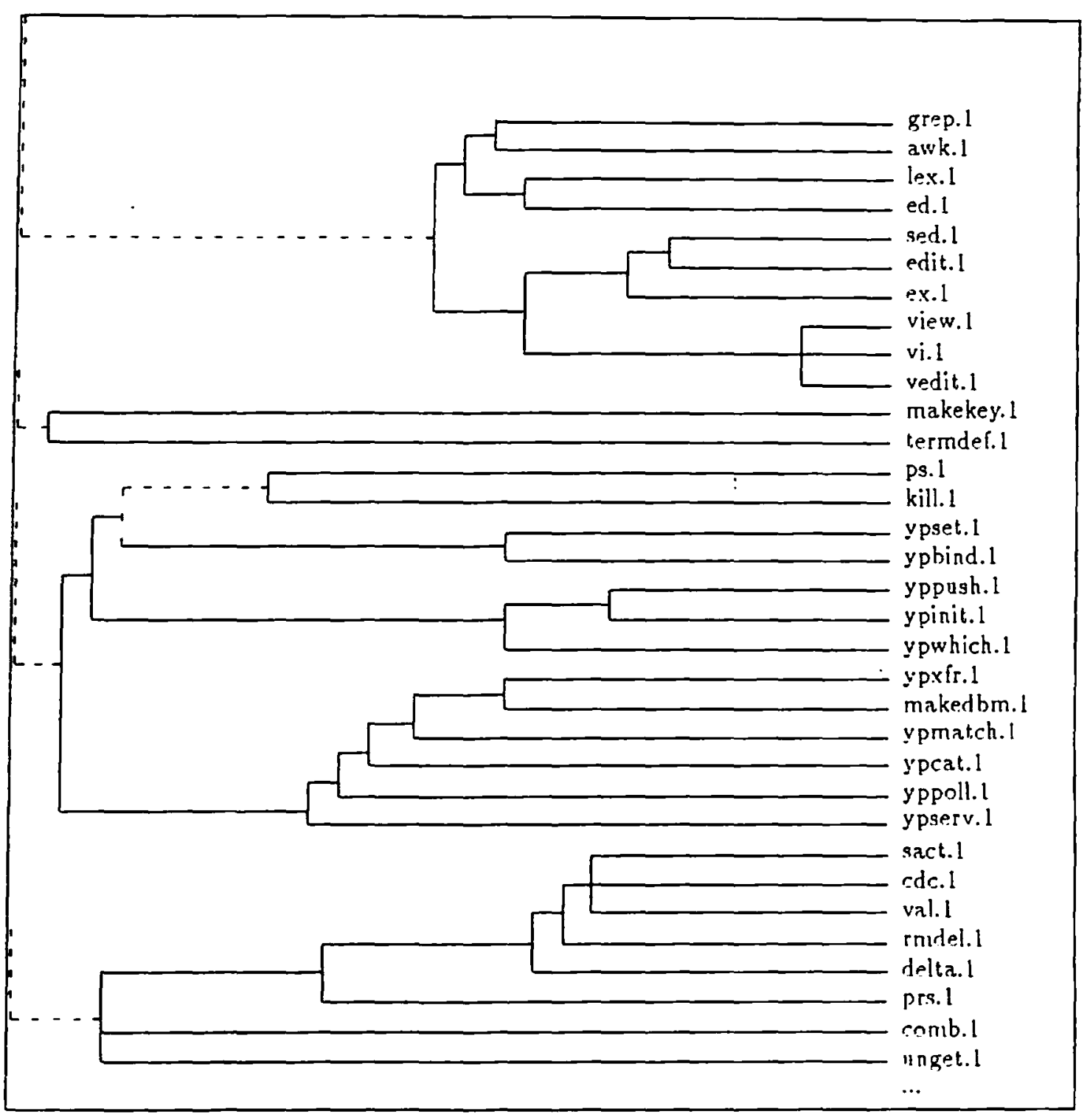

Figure 4: Portion of $\lambda I x$ hierarchy (single link. $k=n .5$ ) 


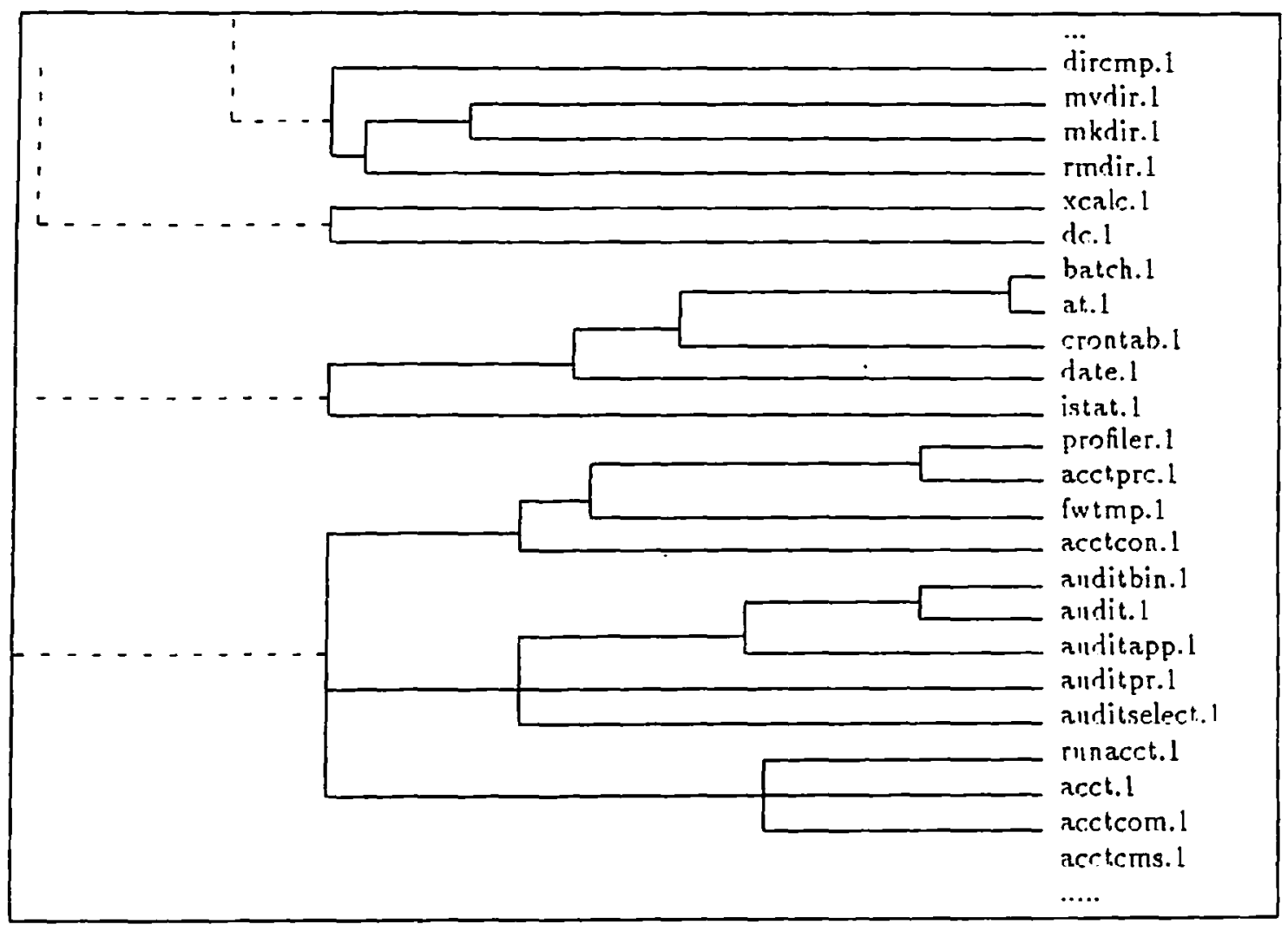

Fignre 5: Portion of Axx hierarchy (single link. $k=0.5$ ) 
- Linear retrieval: A search locates the candidate componenta and the randidates are ranked arcording to their degree of match with the query.

- Browsing Cluster-based retrieval is initiated when no adequate components have been lound by the linear retrieval.

The following explains how these three stages are supported in our approach.

\subsection{Query specification}

Using uncontrolled-vocabulary indexing as we do presents clear advantages at the query specification stage. Indeed, a minimum of constraint is pilt on the user as $s /$ he expresses her/his query. The user does not have to learn a specific index language or understand the organization of the library. S/he can express her/his query in natural language and then the indexing component is applied in order to tiranslate the query into attributes nnderstandable by the system. Exactly the same terhnique is used for extracting LAs from natural-langnage queries as from natural-language documentation. This provides a very convenient and user-friendly interface between the user and the library system. berallse the user is not constrained by any rigid formalism.

The queries can be expressed in free-style natural language. However, the user must be aware of the fact that queries are not, really interpreted, but rather considered as a description of the functionality of the desired component. For instance, the user could express queries of the form "How can I do such and such" since only the "such and such" would be considered for indexing, the rest, heing either closed-class words or words with low quantity of information. Formulating a query that neressitates some understanding, such as a query including negations like "but not", would only lead to wrong interpretation. Let ns note that it wonld be possible at this point to allow some simple interpretation of the queries, by allowing for instance the usual boolean connectors ("and", "or". "but not"). This wonld clearly boost the performance of the library system. However, since on point here is to show how far we can go without understanding either the queries or the documents, we do not discuss these possible enhancements.

\subsection{Linear retrieval}

In order to retrieve the best candidates for a given query, we apply the usual IR. method, which consists of ennsidering the query as a docminent and retrieving the component.s in the repository whose signature are the most similar to the signature of the query. $\Lambda$ possible measure of similarity is the $\delta$ measure defined in (1.3, Section 4.2.2. The most similar components are then returuerl th the user, ranked in order of derreasing similarity with the query. The linear retrieval technique is promintod in Fignre 6.

In case of low recall, that is, if the user is not satisfied with the retrinvord candidates, a more fuzay search can be performed that also considers partial matching LAs. In that case nnly L, As that partially match a query LA, i.e., have one word in common, are considered. This signifirantly inrreases the recall but as a tradeoff drastically decreases the precision. It should therefore he usad only when the user considers that nothing relevant lias been retrieved with the initial query. An example of linear retrieval is given in Figure $i$.

In Figure 7 , the candidates are ranked in order of decreasing similarity with the query ("How can I locate regular expressions in a file"). Therefore, the top candidates nsually answer the query the best. In the example shown in Figure 7 , all the candidates retrieved deal more or less strongly with regular 
Get natural-language query from user

Index query and produce its signature $Q=\left\{\left(w, w^{\prime}, \rho\right)\right\}$

For each query $L A,\left(w, w^{\prime}, \rho\right) \in Q$

$C\left(w, w^{\prime}\right)-\left\{c \mid \exists \rho\right.$, sich that $\left.\left(w^{\prime}, \rho, c\right) \in \lambda(w)\right\}$

(i.e., identify all the components that have this $I, A$ in their signature) EndFor

$C-\cup\left\{C\left(w, w^{\prime}\right)\right\}_{(w, w, w) \in Q}$

For each $c$ in $C$

Evaliate the similarity between the signature $S_{c}$ of $c$ and $Q$ as $\delta\left(Q, S_{c}\right)$ (where $\delta$ is the similarity measure defined in (13))

Rank components in order of decreasing similarity.

Figure 6: Linear retrieval technique

Proressing query:

How can I locale a regular espression in a file

Lemmatizing sentence...

Searching...

regex.3220.21

regexp.3 220.21

awk.l $\quad 7 \pi .32$

grep.1 $\quad 77.32$

find.1 33.88

ogrep.1 $28.7 T$

regemp.3 28.71

dosfirst.3 22.38

dosnext.322.38

Figure 7: Example of linear retrieval 
Processing query:

Establish a new password.

Lemmatizing sentence...

Searching...

passwd.1 32.05

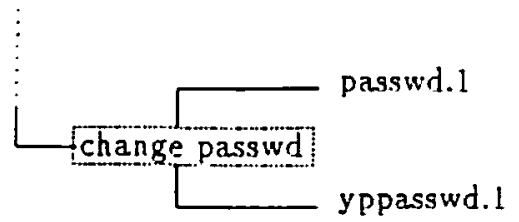

Linear retrieval

Cluster-based retrieval

Fignre 8: Browsing in the hierarchy

expressions. Even the two last candidates, dosfirst and dosnext, do not, answer the query. bit are very slightly related since they allow locating DOS files that match a pattern.

\subsection{Browsing, cluster-based retrieval}

The retrieval stage in classical library management systems is often limited to lorating a set of components exactly matching the user's query or, when such components do not exist. related components. Library systems do not usually provide any further assistance, whereas many IR systems do.

In our approach, the user may communicate interactively with the system in order to direct the browsing when $s /$ he is not satisfied with the first retrieval yielded. The linear search retrieves the most related candidates, and then the browsing process begins.

Typirally, the user starts from one of the candidates retrieved hy the linnar search and explores the hierarchy bottom-up. Consider the browge hierarchy given in Figure 4 and suppose that a user gives a query asking about ways "to identify a process". If the first candidate retrieved at the retrieval search is $k$ ill. Then, the user can access the browse hierarchy, and explore the rlusters including $k i l l$ in order to determine which components are strongly related. In onr example, $s /$ he will find $p s$ as the most related component, which is clearly a better candidate for this given query than the one retrieved by the linear search. Anuther example is illustrated in Figure 8. The two relevant randidates in Aix for the query "establish a new password" are passud and yppassud. However, the linoar retrieval retrievea only passud simply because the query had no intersection with the signature of yppassud. $\lambda t$ this point. the user could reformulate the query, but s/he might not be aware that s/he lias missord some relevant, candidates. Uaing the browse hierarchy is therefore more convenient, in order in rhork if snme unexpecterl candidates have been missed. In the example, both passud and yppassud are atinngly rolaterl: their signatures share the LA (change passud), and therefore belong to the same low-Invel cluster in the browsing hierarchy. Browsing in the hierarchy from passod allows the nser to retrieve the other relevant candidate. These two examples show how a browse hierarchy can help improve the finding of possihle candidates that could be missed via linear retrieval.

At any point, the user can consult the signature of a component in order to have more information about its functionality. Fast access to signatures is achieved via the signature repository. The user can also provide, at any stage, fnrther information in order to get a finer ratrieval. By browsing, s/he gets

\footnotetext{
"Note that "passud" here is proper name and is different from the noun "password" montioned in the query.
} 
more information about components and learns how to provide disrriminating queries.

\section{Empirical Results}

The approach described in the previons sections has been embodied in a tool. Gusu, which has been fully implemented. partly in VSPascal and partly in C, under AIX. The system has reached a satisfactory first. stage, and the implemented version yields quality results.

We have tested our system on the entire AIx documentation available to 11s, which describes approximately 1,100 AIX components. When brilding the index repository. we therefore processed the entire documentation that forms a corpus of more than 800,000 words, and we identified 18,000 LAs for the 1,100 signatures.

In order to evaluate Guru's performance, we used the following criteria.

- User effort. This consists of all the effort that mist be expended by the nser in order to nse the library system. It is impossible to formally measure user effort. However, thanks to the uncontrolled vocabulary approach that we applied, we believe that the effort, that must be invested for nsing Gusu is minimal. Queries can be formulated in natural language, and therefore the user is not required to learn any index language and formalism.

- Maintenance effort. This consists of all the effort that is neressary to keep the system working and up to date. This effort includes, in particular, indexing new components and adding them to the library. The maintenance stage is highly facilitated in Guru. The indexing is performed antomatically and the insertion of new components can be done incrementally. Kaplan and Maarek, in [20], have proposed several algorithms for incrementally "pdating a repository of LA-based indices when ingerting, deleting or modifying components.

- Effciency. This refers to the average interval between the time a query is issued and the time an answer is given. Efficiency becomes an issue only if a rel,rirval takes so long that nsers start to complain. Our experience with the system shows that efficiency is not, an issue, as the response time is reasnnable. Profiling the execution of the query program showed that the time to periorm the query was dominated by the time to map the repository file in to the address space of the query program. The lookup operations and the printing of the LA-file name pairs consumed almost no time in enmparison. Test queries involving from 5 to $15 \mathrm{LAs}$ each took approximately 2.5 seconds on an RT, and 0.15 seconds on an IBM RISC System/fnnn. The hetter performance of the latter is partly due to its more efficient implementation of file mapping.

- Retrieval effectiveness. This is rlearly the most important performance rriterion. It refers to the system's ability to provide information services as needed by the nser.

The next section focuses on evaluating the retrieval effectiveness of Cirnus. 


\subsection{Measuring Retrieval Effectiveness}

\subsubsection{Recall and Precision}

The most widely used measures for evaluating retrieval effectiveness are recall and precision [32]. Recall is defined as the proportion of relevant material, i.e., it measures how well the considered system retrieves all the relevant components. Precision is defined as the proportion of retripved material that is relevant, i.e., it measures how well the system retrieves only the relevant components. Recall can also be interpreted as the probability that a relevant component will be retrieved, and precision as the probability that a retriever component will be relevant [5].

Recall and precision can be defined more formally as follows. Let $C:$ be the whole collection of components forming the library. For each query, $C$ can be partitioned in to two disjoint sets, $R$, the set of relevant material and $\bar{R}$ the set of irrelevant material. Given the query, the system retrieves a set of components $c$ that can also be partitioned into relevant and irrelevant material, respectively, $r$ and $\bar{r}$. Recall and precision are defined as:

$$
\begin{aligned}
\text { recall } & =\frac{T}{R} \\
\text { precision } & =\frac{r}{c}
\end{aligned}
$$

Recall and precision measurements require the ability to distinguish between relevant and irrelevant material. For relatively small collections such as the AIX collection, it is possible to manually determine the set of relevant material for a given query.

\subsubsection{Experiments and Comparison}

This section describes the experiments that allowed us to evaluate the retrieval effectiveness of Guru. As a hasis for comparison, we have considered INFOEXPLORER, which is an IRN RISC: System/6000 CD.Rom Hypertext Information Rase Library. INFOF,XPLORER is a recent hypertext system that given access to the dosumentation for $A \mathrm{IX}$ and for associated programs. INFOEXPI,ORER provides not only hypertext links between pieces of the dix documentation, but also search and retrieval farilities based on state-of-the-art IR techniques. Queries can be expressed as single word search or multiple word compound search with no control of vorabulary. The componnd search, which is the most plahorated, allows the nser to express a query as a word pattern formed of single words related by thron pnssihlo mnnertors, "and". "or", and "butnot". Moreover, the user can restrict the search. S/he can givo rnnstrnints specifying if the pattern words must appear within the same article or within the same paragraph, the proximity of these words within a paragraph, and the search fields and the search categnrios.

When given such a query. INFOEXPLORER returns a list of randidalos that exactly fit the query, ranked according to the frequency of the pattern in the considered loriment. No signature is built for the documents examined: all words appearing in the text are considpred during search. Therefore. INFOEXFLCRrR can be expected to have a a much higher recall but lower precision than GURU. We do not need to also compare efficiency, i.e., retrieval speed. Gusul is, independently of implementation, much faster than INFOEXPLORER since it does not explore the entire textual database but a much smaller repository formed by the signatures.

INFOEXFLORER is thus a quite sophisticated IR tool that represents a good reference for comparison purposes since it is specifically for AIX. Also, INFOEXPLORER encodes a great deal of manually-provided 
information about the stiructure of the documentation. The system has to know about paragraphs, titles, etr.. and thus has been much more expensive to build than Guru. Providing this structural information to our system wonld greatly enhance its performance, but our point here is to show that even without such information, our system can perform nicely thanks to its indexing srheme.

GURU and INFOEXPLORER were compared for retrieval effectiveness. In order to claim this test to be valid, we must fulfill the usual test procednre requirements [32]. These requirements are for

1. the queries to be used for test purposes must be user search requests actually submitted and processed by both systems;

2. the test collection must consist of documents originally included in the library, chosen in such a way that any advance knowledge concerning the retrievability of any given component by either system is effectively ignored; and

3. the number of components considered to be retrieved by the twn systems must be subject to the same cutoff.

To fulfill the first requirement, we have conducted a survey among the graduate students in the Department of Computer Science at Columbia University in November 1988. This survey provided us with a collection of typical queries on (INIX-like systems, as formnlated by ('NIX users ranging from naive users to expert programmers. $\Lambda$ typical query was expressed as a naturaflanguage sentence with an average of 3.7 open class words per query. describing a desired functionality. This kind of query could directly be fed to GURU but not to INFOEXPLORER since the latter's compoind search facility arcepts only boolean queries. Therefore, leefing the queries to INFOEXPLORER required some supplementary effort, first choosing the right connectors between open-class words extracted from the queries, and possibly dropping some words when the recall was too low. In our interaction with the compound search facility, we had to refine and retry the query formulation several times. We kept only the best, result for comparison purposes, since we wanted to compare the tools' indexing schemes rather than their querying facilities. Gunu's querying facility requires less nser effort than INFOEXPLORER's, bnt the latter's rnild be greatly improved if it did not require perfect matches hetween the boolean query and the candidates, using a similarity measure between candidate and query, for instance. The average number of open-rlass words used for questioning INFOEXFI,ORTRR was 3.

As far as the second requirement is concerned. the collection onnsidered for test has been the entire A Ix lihrary. We consulted with several AIX experts at IBM in order to fetermine for earh query the set of existing relevant components in the AIx library so as to be able to evaluate the recall and precision. As our test. collection was composed of about $11 \mathrm{nn}$ components, we selected 30 qurrirs from among all the queries provided by our survey. This ratio corresponds to the same number-of-purrina per number-nf-docnments ratio as the one that has been used in standard test sets surh as Mr.r. (collertion of merdical ahstracts. 30 queries for 1033 documents) or $\mathrm{CISI}^{10}$ (information science alsst.rarts. 35 qurries for 14 fin information abstracts).

As far as the third requirement is concerned, since both systeins ranked the retrieved candidates, we were able to compare rerall and precision at the same ranks.

The comparison was performed by measuring, for both systems, prerision at several levels of recall. We followed the usual procedure [38], [32], which consists of

${ }^{10}$ These test set, have heen used for evaluating aeveral IR systems such as I,SI [9]. 
1. plotting precision-recall curves for each test query with each plot mrresponding to a given elltoff value,

2. extrapolating these cnrves so as to obtain precision values for recall values that were not effectively achieved, and finally,

3. deriving from the enrves computed in stage (2) the average prerision values at fixed recall intervals. so as to obtain a single average precision recall curve for the system considered.

We have built such curves for both Guru and INFoExPLORER and plotted them on the same axes (See Figure 9). The best performance is reached by the system whose curve is closest. to the area where both precision and recall are maximized, the upper right corner of the graph. As mentioned, becallse of the indexing scheme of both systems, we could expect that INFOEXPLORER would achieve a lower precision but higher recall than Cupu. It turned ont that the maximum recall, all ranks included, achieved by both systems "ws approximately the same, around $88 \%$ on the average, bnt, from the graph presented in Fignre 9, it is clear that GuRu had $15 \%$, on the average, better pregision than INFoExPLORER. In other words. Gunu achieves a higher precision withont losing in recall. This is more than satisfactory.

These results clearly prove that we have achieved high precision withont losing recall. The recall rate is significantly increased when we make nse of the Guru browsing facility. For instance, in several cases some related components were not, retrieved during linear retrieval, but only during browsing.

The results of this evaluation should not be seen as the final definitive results, but only as an indicator of what can be expected from a fully operational Guru system. However. even introspective experiments such as those described in this section are sufficient to confirm the advantages of an LA-based indexing scheme. Onr LA-based indexing scheme makes the indexing language exhanstive as weil as specific and thus ensures good retrieval performance. The experimental results confirmed the expectations an can be considered as encouragements to pursue research in the direction pursued in this paper.

\section{Conclusion}

We have presented a method for automatically constructing software libraries from a collection of documented but unindexed software components. We discussed the advantages of nsing natnral-language docimentation as opposed to source code, assnming any documentation is available, as a source of functional information. We then described a new free-text indexing scheme, for alltomatically producing document signatures, based upon a richer unit than single terms, the lexical anffinity. All associated software components conld then be classified, stored, compared and retrieved, via linear nr cluster-based terhniques, arenrding to these indices.

These methods and schemes are embodied in a new tool whirh has hron implemented and evaluated for retrieval effectiveness. The evaluation compared Guru with the INFOF,xri,orer hypertext library, built specifically to help find soltware components in the AIx system. The average recall-precision cnrves of both tools were computed. The results of this test indicate that Curnts performance was better than INFOF.XPLORF, This result is very enconraging since INFOEXFLORFR was murh more expensive to build and specifically tailored to the AIX library.

The major contribution of this work consigts of bringing classical and new in lormation retrieval techniqnes to bear in software reuse. This involved:

- Designing a new indexing scheme based on high information eontent lexical affinities. 


\begin{tabular}{|l|l|l|l|}
\hline Recall & GURU precision & INFO precision & Improvement \\
\hline \hline 0.1 & 0.85 & 0.7 & $15 \%$ \\
0.3 & 0.84 & 0.68 & $15 \%$ \\
0.5 & 0.76 & 0.56 & $20 \%$ \\
0.7 & 0.58 & 0.4 & $18 \%$ \\
0.9 & 0.52 & 0.39 & $13 \%$ \\
\hline
\end{tabular}

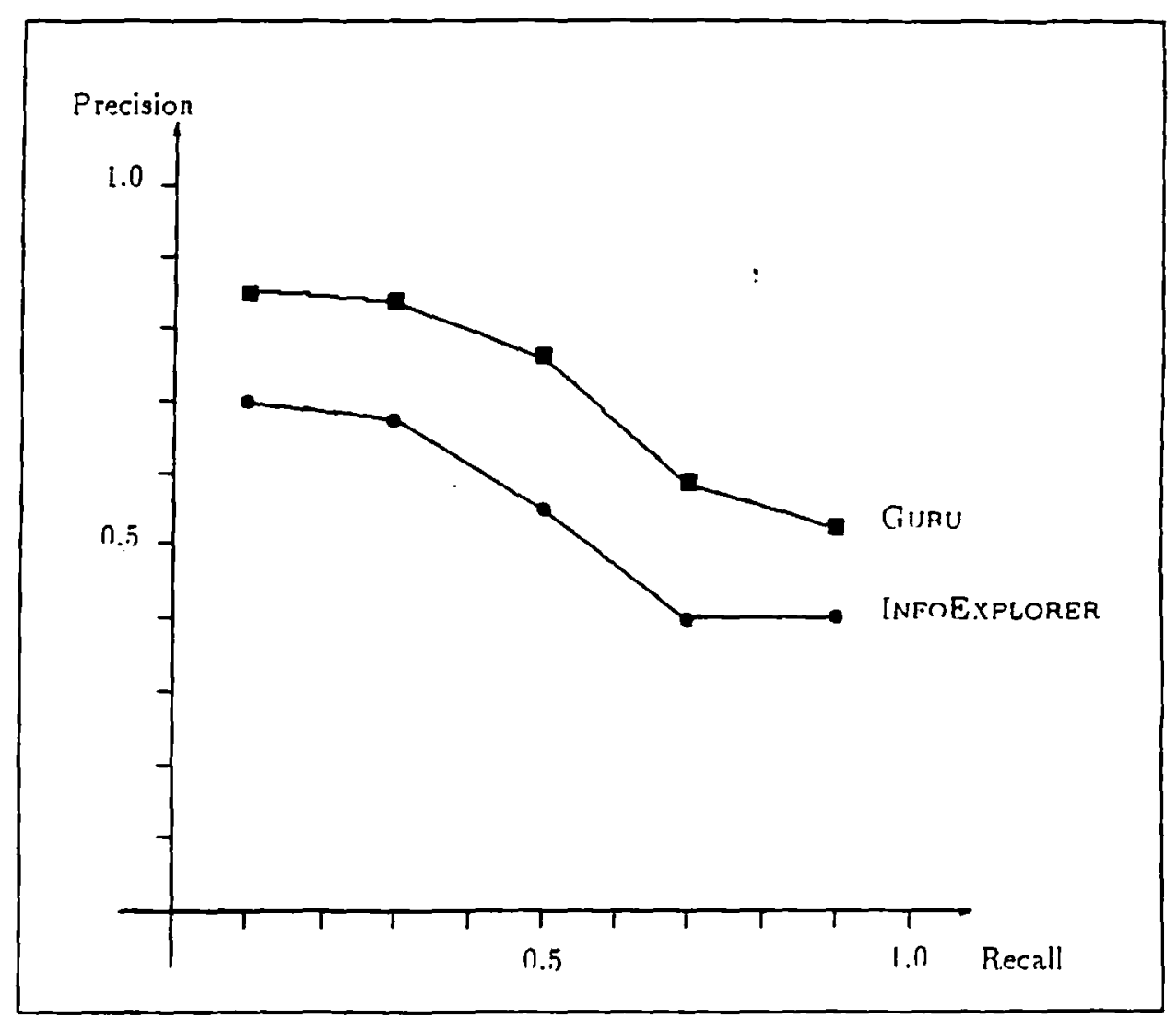

Fignre 9: Precision-recall curves (means across quering) 
- Alapting classical numeriral rlister analysis techniques for assembling software romponents into browse hierarchies.

- Designing retrieval merhanisms sperifically adapted to the LA-hased indexing scheme so as to provide a rompiete storage anji retrieval framework.

Finally, the evaluation we have performed seems to indicate that Salton's statement. abont the limitation of the "phrase generation" approach in indexing (See Section 3.1) is overly pessimistic and that significant improvements over single terms techniques can be achieved at relatively lnw cost..

\section{Acknowledgments}

Y. Marek performed part of this work while at the Technion, Department of Computer Science, Haifa, Israel, partly sllpported by a Gutwirth Fellowship. G. Kaiser is supporter by National Science Fonndation grants CDA-8920080, CCR-8858029 and CCR-880274l, by grants from AT.ET. BNR. Citicorp. DEC. IBM. Siemens. Sun and Xerox, by the Center for Advanced Technology and by the Center for Telecommunications Research.

We would like to thank Mark Kennedy who helped a lot in the design and implementation of GuRu's retrieval component.

\section{References}

(1) M. Adanson. Histoire Naturelle du Sénegal. Coguillages. Auec la relatinn abrégèe d'un voyage fait en ce pays. pendant les années 1749,50.51.52 et 5.3. Bauche. Paris. France, 1757.

[2] B.P. Allen and S.D. Las. A knowledge-based environment for the development of software parts composition systems. In Prnceedings nf the $11^{\text {th }}$ ICSE. pages 104-112. Pittshurgh. PA, May 1989.

[3] S.P. Arnold and S.I. Stepoway. The reuse system: Cataloging anrl retrieval of reusable soltware. In W. Tracz, oditor. Snftunre Reuse: Emerging Technolngy, pages 138-141. Computer Society Press, 1987.

[4] R. Ash. Informalion Thenry. Interscience Publishers (John Wiley it Snns), New York, 1965.

[5] D.C. Blair and M.E. Maron. An evaluation of retrieval effectiveness inr a full-text dornment retrieval system. Communications of the ACH, 28(3):289-299. March 1985.

(6) B.A. Burton, R. Wienk Aragon, S.A. Bailey, K.D. Koelher, and I.A. Mayeg. The railsable software library. In W. Tracz, editor. Snfturare Reuse: Emerging Techunlngy. pagea 129-13i. (:ninputer Society Press, 1987.

[i] F. Can and E.A. Ozkarahan. A clustering scheme. In Prncredings of SIGIR'R.3, pages 115-121. Bethesda, MD. June 1983. ACM Press.

[8] F. de Sallssure. Cours de Linguistique Générale, Quatrième Editinn. Tihrairie Payot, Paris, France, 1.949.

[9] S. Deerwester, S.T. Dumajs, G.W. Furnas. T.K. Landaner, and R. Marshman. Indexing by latent semantic analysis. Journal nf the American Sociely for Informalinn Srience. $11(6): 391 \cdots 407,1990$. 
[10] P. Devanbu. Re-use of software knowledge: A progress report. In Third Annual Workshop: Methods and Tonls for Reuse, Syracuse, NY. June 1990.

[11] P. Devanbu, P.G. Selfridge. B.W. Ballard, and R.J. Brachman. A knowledge-based software information system. In Prnceedings of IJCAI89, pages 110-115. Detroit. MI, August 1989.

[12] E. Diday, J. Lemaire, and F. Testu. Elements d'dnalyse des Données. Dunod, Paris, France, 1982.

[1.3] B. Everitt. Cluster Analysis. Halsted Press (John Wiley \& Sons), New York, 1980.

[14] W.B. Frakes and P.B. Gandel. Classification, storage and retrieval of reusable components. In N.J. Belkin and C.J. van Rijsbergen, editors. Proceedings of SIGIR'89. pages 251-254, Cambridge. MA, June 1989. ACM Press.

[15] W.B. Frakes and B.A. Nejmeh. Software reuse through information retrieval. In Proceedings of the 20th Annual HICSS, pages 5.30-535, Kona, HI, January 1987.

[16] A. Griffiths, L.A. Robinson, and P. Willett. Hierarchical agglomerative clustering methods for antomatic document classification. Journal of Documentation, 40(3):175-205. September 1984.

[17] W. Harrison. A program development environment for programming by refinement and reuse. In Proceedings of the 19th HICSS, pages 459-469, Kona. HI, 1986. CS Press.

[18] T. Ichikawa and M. Hirakawa. Ares: A relational database with the capability of performing flexible interpretation of queries. IEEE Transactions of Snftware Engineering, 12(5):624-634, May 1986.

[19] N. Jardine and C.J. van Rijsbergen. The use of hierarchic clustering in information retrieval. Informalinn Slornge and Retrieunl, 7(5):217-240, December 1971.

[2ij] S.M. Kaplan and Y.S. Maarek. Incremental. maintenance of semantic links in dynamically changing hypertext systems. Interacting with Computers, December 1990. In press.

[21] P.H. Klingbiel. Machine-aided indexing of technical literature. Infnrmalinn Storage and Retrieyal, 9:79-84, 1973.

[22] G.N. Lance and W.T. Williams. A general theory of classificatory sorting strategies. Conmputer Journal, 9:37.3-38ก, 1967.

[23] M. Luhn. The antomatic creation of literature abstracts. IBM Jnurnal n/ Research and Development. 2(2):159-165, April 1958.

[21] Y.S. Maarek. Using Struclural Information for Managing I'ery Large Snfluare Systems. PhD thesis, Technion, Israel Institute of Technology, Haifa, Israel, January 1989.

[25] Y.S. Maarek. An incremental conceptual clustering algorithm with input-ordering bias enrrection. In M.C. Golumbic, editor, Advances in Artificial Inlelligence. Natural Language and Knnwledge Base Systems. Springer Verlag, 1990.

[26] Y.S. Maarek and G.E. Kajser. On the use of conceptual clustering for classifying rellsable ada code. In Ada letters, Using Ada: ACM SIGAda International Conference, pages 208-215. Boston, MA, December 1987. ACM Press.

[27] Y.S. Maarek and F.A. Smadja. Full text indexing based on lexical relations. an application: Software libraries. In N.J. Belkin and C..J. van Rijsbergen, editors, Proceedings nf SIGIR'89, pages 198-206, Cambridge, MA, June 1989. ACM Press. 
[28] W.J.R Martin. B.P.F. Al, and P.J.G. van Sterkenburg. On the processing of a text corpus: From textnal data to lexicographic information. In R.R.K. Hartmann, editor, Lexiengraphiy: Principles and Practice, London, 1983. Applied Language Studies Series. Academic Press.

[29] R. Michalski and R. Stepp. Aıtomated constructions of classifications: Conceptual clustering versus numerical taxonomy. IEEE Transactions an Pattern Analysis and Machine Intelligence. 5(4):396-409, Jily 1983.

[30] R. Prieto Diaz and P. Freeman. Classifying software for reusability. IEEE Software, 4(1):6-16, Jannary 1987.

[31] G. Salton. Automatic text processing, the transformation, analysis and retrieval of information by compister. Addison-Wesley, Reading, MA, 1989.

[32] G. Salton and M.J. McGill. Introduction to Modern Information Retrienal. Computer Series. McGrawHill. New York. 1983.

[33] G. Salton and M. Smith. On the application of syntactic methodologies in automatic text analysis. In Proceedings of SIGIR '89, pages 137-150. Cambridge, MA, June 1989. ACM Press.

[34] R.W. Schwanke, R.Z. Altncher, and M.A. Platoff. Discovering, visualizing and controlling software structure. In Proceedings of the Fifth International Workshop nn Snftuare Specifications and Design. pages 147-150. Pittsburgh, PA, May 1989.

[35] F.A. Smadja. Lexical co-occurrence: The missing link. Journal of the Association for Literary and Linguistic Computing. Oxford University Press, 4(3). 1989.

[36] K. Sparck Jones and J.I. Tait. Antomatic search variant generation. Journal of Documentation, 40(1):50-66, March 1984.

[37] W.F. Tichy, R.L. Adams, and L. Holter. NLH/E: A natural-language help system. In Proceedings of the $1 l^{\text {th }}$ ICSE, pages 364-374, Pittsburgh, PA, May 1989.

[38] C.J. van Rijsbergen. Informalion Retrielal. Butterworths, second erlition, 1979.

[39] M. Wond and I. Sommerville. An information retrieval system for soltware components. SIGIR Forım, 22(3.4):11-25, Spring/Summer 1988. 